\title{
Adaptive Dynamics and Evolving Biodiversity
}

\author{
Ulf Dieckmann and Régis Ferrière
}

\subsection{Introduction}

Population viability is determined by the interplay of environmental influences and individual phenotypic traits that shape life histories and behavior. Only a few years ago the common wisdom in evolutionary ecology was that adaptive evolution would optimize a population's phenotypic state in the sense of maximizing some suitably chosen measure of fitness, such as its intrinsic growth rate $r$ or its basic reproduction ratio $R_{0}$ (Roff 1992; Stearns 1992). On this basis it was largely expected that life-history evolution would always enhance population viability. In fact, such confidence in the prowess of adaptive evolution goes back as far as Darwin, who suggested "we may feel sure that any variation in the least degree injurious would be rigidly destroyed" (Darwin 1859, p. 130) and, in the same vein, "Natural selection will never produce in a being anything injurious to itself, for natural selection acts solely by and for the good of each" (Darwin 1859, p. 228).

The past decade of research in life-history theory has done away with this conveniently simple relation between population viability and evolution, and provided us with a picture today that is considerably more subtle:

- First, it was realized the optimization principles that drive the evolution of life histories could (and should) be derived from the population dynamics that underlie the process of adaptation (Metz et al. 1992, 1996a; Dieckmann 1994; Ferrière and Gatto 1995; Dieckmann and Law 1996). In the wake of this insight, the old debate as to whether $r$ or $R_{0}$ was the more appropriate fitness measure (e.g., Stearns 1992; Roff 1992) became largely obliterated (Pásztor et al. 1996).

- Second, we now understand that the particular way in which population densities and traits overlap in their impact on population dynamics determines whether an optimization principle can be found in the first place, and, if so, what specific fitness measure it ought to be based on (Mylius and Diekmann 1995; Metz et al. 1996b). It thus turns out that for many evolving systems no optimization principle exists and that the conditions that actually allow the prediction of life-history evolution by maximizing $r$ or $R_{0}$ are fairly restrictive (e.g., Meszéna et al. 2001; Dieckmann 2002).

- Third, it became clear that, even when adaptive evolution did optimize, the process would not necessarily maximize population viability (Matsuda and Abrams 1994b; Ferrière 2000; Gyllenberg et al. 2002; Chapter 14). In addition, it has been shown recently that, even when adaptive evolution gradually 
improves population viability, such a process could eventually lead to a population's sudden collapse (Renault and Ferrière, unpublished; Parvinen and Dieckmann, unpublished).

This chapter expounds in detail the intricate link between adaptive evolution and population viability. Section 11.2 reviews conceptual limitations inherent in the traditional approaches to life-history evolution based on optimization criteria, and Section 11.3 introduces adaptive dynamics theory to overcome these limitations. Adaptive evolution without optimization has intriguing consequences for the origin and loss of biodiversity, and these implications are reviewed in Sections 11.4 and 11.5 , respectively. While the processes described there can unfold in a constant environmental setting, Section 11.6 provides an overview of how the viability of adapting populations can be affected by environmental change.

\subsection{Adaptation versus Optimization}

Life-history optimization in the form of maximizing $r$ or $R_{0}$ has been applied widely to a variety of questions in evolutionary ecology, including the evolution of clutch size, age and size at maturation, sex ratio, reproductive systems, and senescence. Unfortunately, however, this approach faces several fundamental limitations. Since these restrictions are conceptually important and have wide-ranging significance for evolutionary conservation biology, we discuss them in some detail, before, in the next section, summarizing a framework with which to surmount the difficulties.

\section{Optimization in earlier evolutionary theory}

Despite repeated discussions about the limitations of optimizing selection (e.g., Lewontin 1979, 1987; Emlen 1987), it is surprising how long it has taken to account thoroughly for these limitations in the practice of evolutionary ecology research - to the extent that this process is still ongoing today. We thus start out with a brief sketch of some key earlier approaches that favored the idea of evolution as an optimizing process:

Following a notion introduced by Wright (1932) early on in the modern synthesis, adaptive evolution is often envisaged as a hill-climbing process on a fixedfitness landscape. Whereas Wright originally considered adaptive landscapes based on the dependence of mean population fitness on genotype frequencies, subsequent work extended Wright's concept by utilizing adaptive landscapes to describe the dependence of individual fitness on phenotypes. Yet, Wright himself recognized that the adequacy of his convenient metaphor was lost when selection was frequency dependent (Wright 1969, p. 121).

The same conclusion applies to Fisher's so-called "fundamental theorem of natural selection" (Fisher 1930). This predicts mean population fitness to increase monotonically over the course of adaptive evolution - provided, however, that certain restrictive assumptions are fulfilled. It is not surprising that one of these assumptions is the constancy of fitness values, and thus the absence of 
frequency-dependent selection (Roughgarden 1979, p. 168; Frank and Slatkin 1992). To reconcile this assumption with the fact that, in the long-term, the mean absolute fitness of a population must hover around zero, Fisher stipulated a balance between the "progress" of natural selection and a "deterioration" of the environment: "Against the rate of progress in fitness must be set off, if the organism is, properly speaking, highly adapted to its place in nature, deterioration due to undirected changes either in the organism [mutations], or in its environment [geological, climatological, or organic]" (Fisher 1930). The quote illustrates that when explaining the environment's "deterioration" Fisher did not appear to have thought of density- or frequency-dependent selection. Today, evolutionary ecologists realize that a phenotype possessing a relative fitness advantage when rare loses this advantage once it has become common. As we show below, the infamous environmental deterioration simply results from a changing composition of the evolving population itself. Therefore, densityand frequency-dependent selection are at the heart of reconciling the conflict between Fisher's theorem and long-term population dynamics.

- Also, the fitness-set approach developed by Levins (1962a, 1962b, 1968) still enjoys widespread recognition in life-history evolution (Yodzis 1989, pp. 324351; Calow 1999, p. 758; Case 1999, pp. 175-177). It is based on the assumption that, within a set of feasible phenotypes defined by a trade-off (the "fitness set"), evolution maximizes fitness (referred to as the "adaptive function" by Levins). Since the adaptive function is assumed to remain constant in the course of evolution, selection is optimizing and frequency-dependent selection is excluded.

- Results presented by Roughgarden (1979) overcame the strict confines of selection on fixed-fitness landscapes. Yet Roughgarden's approach to adaptive evolution by maximizing a population's density is applicable only when selection is density dependent, and not when it is frequency dependent.

- The concept of frequency-dependent selection also continues to receive short shrift in contemporary textbooks on life-history evolution. For example, out of the 465 pages of Roff (2002), not more than five deal with the description and implications of frequency-dependent selection, while the corresponding percentage in the seminal textbook by Stearns (1992) is even smaller.

We now proceed with a detailed review of the reasons that preclude the application of optimality principles to realistic problems in evolutionary ecology. Complementary to the considerations below are long-standing debates about the roles of developmental constraints (e.g., Maynard Smith et al. 1985) and of accidental historical by-products of evolution (e.g., Gould and Lewontin 1979) in obscuring the match between observed evolutionary outcomes and underlying "fitness maxima".

\section{The quest for suitable optimization criteria}

Even evolutionary biologists who favor optimality approaches concede that it is not always obvious which specific optimization criteria ought to be applied. In particular, the results of maximizing $r$ or $R_{0}$ usually are not equivalent. For instance, 
predictions about the evolution of reaction norms for age and size at maturation critically depend on whether $R_{0}$ (Stearns and Koella 1986) or $r$ (Kozlowski and Wiegert 1986) is used as the optimization criterion. Consequently, the question as to which function should be viewed as the Holy Grail of fitness measures has led to heated debate, reviewed, for example, in Roff (1992), Stearns (1992), Charnov (1993), and Kozlowski (1993).

The key issue here, recognition of which resolves the earlier debate for good, is that the bi-directional interaction between an evolving population and its environment was missing from the discussion (Metz et al. 1992). Whereas few biologists would contest that fitness always depends both on an individual's phenotype and on the environment the individual experiences, classic fitness measures used as optimization criteria, like $r$ or $R_{0}$, only capture the former dependence. From today's perspective it is self-evident that the drastic reduction in complexity implied by dropping from consideration the dependence of fitness on the environment can only be justified under rather restrictive conditions. In particular, this convenient simplification is warranted only if the environment of an evolving population stays fixed, instead of varying along with the evolutionary change. Most of the time, however, conspecifics form an integral part of the environment that individuals experience. Therefore, when the distribution of conspecific phenotypes changes, so does a focal individual's environment. This explains why to maximize classic fitness measures like $r$ or $R_{0}$ cannot do justice to the richness of phenomena in life-history evolution.

\section{Optimization arguments in evolutionary game theory}

The crucial importance of envisaging fitness as a function of two factors, an individual's trait(s) and its environment, was highlighted early on by work in evolutionary game theory (Hamilton 1967; Maynard Smith and Price 1973; Maynard Smith 1982). The payoff functions employed in that approach, which depend on two (usually discrete) strategies, and the broader notion of feedback between an evolutionary process and its environmental embedment are linked because, at ecological equilibrium, a population's resident strategies determine crucial aspects of its environment. When characterizing fitness we can therefore often simply replace a set of environmental variables by a description of the trait values currently resident in the population, and thus arrive at the notion of strategy-specific payoffs in which the explicit consideration of environmental variables is suppressed.

With regard to optimization arguments in evolutionary game theory, some confusion has arisen over two important distinctions: one between local and global optimization, and another between particular and universal optimization. An evolutionarily stable strategy (ESS) is essentially defined as one that maximizes payoffs in the environment the ESS sets for itself, and thus it adopts a global, but particular, notion of optimization. First, alternatively an ESS can be construed locally as a strategy that cannot be invaded by any neighboring strategy, a notion that is especially relevant when quantitative characters or metric traits are considered a ubiquitous situation in life-history evolution. Second, it is crucial to understand 
that an ESS obeys a particular, and not a universal, optimization principle: the ESS usually maximizes payoffs only in its own environment, and not in the many other environments set by alternative resident strategies. This is a significant restriction, since, unless the ESS is already known, it thus cannot be recovered from this particular optimization principle (Metz et al. 1996b). Again, it is therefore only under restrictive conditions that an ESS maximizes payoffs in some "standard" environment that is independent of which phenotype is currently prevalent in the population and can be applied universally throughout the evolutionary process. And it is only in still more restrictive cases that such an optimization criterion happens to coincide with maximizing $r$ or $R_{0}$ (Box 11.1).

\section{Limitations to the existence of optimization criteria}

The preceding discussion shows that it is by no means clear that for a given system an optimization principle exists. Whether or not such a principle can be found critically depends on how an evolving population interacts with its environment. This interaction is characterized by what we refer to as the eco-evolutionary feedback loop. To describe this feedback loop involves specifying the genetically variable and heritable traits, their impact on the focal organism's life history, and the ecological embedding that determines how life-history traits affect and are affected by environmental conditions.

It turns out that when one departs from the simplest ecological embeddings (e.g., the case in which the effect of density dependence is equally felt by all individuals in a population, irrespective of their phenotypes) optimization criteria cease to exist. It can even be shown that this is always the case if the "dimension" of the eco-evolutionary feedback loop is larger than one, a situation that readily arises in many realistic models and implies that populations are experiencing frequency-dependent selection (Heino et al. 1997b, 1998; Box 11.1). From a mathematical point of view, the conditions under which an optimization criterion exists are clearly degenerate (Metz et al. 1996b; Heino et al. 1997b), with the technical term "degenerate" meaning "infinitely rare". This finding contrasts rather sharply with the widespread use of optimization arguments in current evolutionary ecology. It may well be that a limited perception of the range of feedback scenarios actually existing in nature biases our evolutionary models toward the simple subset that conveniently obey optimization principles (J.A.J. Metz, personal communication). In particular, while frequency-dependent selection is still treated as a special case by virtually every contemporary textbook on evolution, this mode of selection is increasingly being recognized as one that ubiquitously acts on many life-history traits involved with, for example, foraging or reproduction (e.g., Kirkpatrick 1996). Since optimization approaches are invalidated by all (non-trivial) forms of frequency-dependent selection (Heino et al. 1997b), the absence of optimization criteria from realistic models of life-history evolution must be accepted as the rule, rather than the exception. 
A celebrated example of an evolutionary game in which no single quantity can be construed as being maximized by evolution is the rock-paper-scissors game (rock beats scissors by crushing, paper beats rock by wrapping, scissors beat paper by cutting). The intransitive dominance relation in this game has been used to explain the coexistence of three mating strategies - "territorial", "mate-guarding", and "sneaking" - in the sideblotched lizard Uta stansburiana (Sinervo and Lively 1996; Sinervo et al. 2000). In that system the population growth rate of each strategy was shown to depend on the composition of the established, or resident, population, in such a way

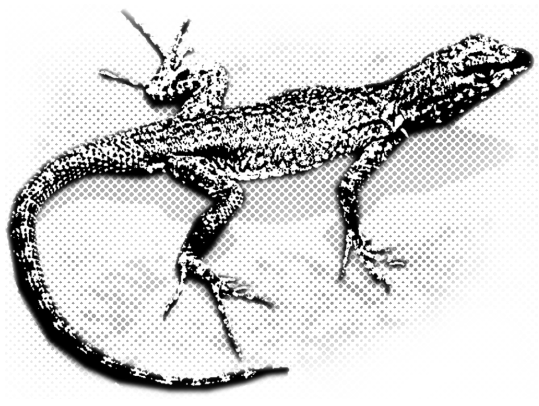

Side-blotched lizard Uta stansburiana that the territorial strategy beats the mate-guarding strategy in an environment where mate-guarding is prevalent, while the mate-guarding strategy wins against sneakers in the environment set by sneakers, and sneakers beat territorials in the environment set by territorials. In cases like this, characterized by the absence of an optimization principle, the study of life-history evolution must rely on evaluating which sequences of invasion are possible, and to which evolutionary outcome they lead.

\section{Evolutionary stability and attainability}

Classic evolutionary game theory, as well as approaches of $r$ or $R_{0}$ maximization, are based on the assumption that phenotypes predicted to be unbeatable or evolutionarily stable against all other possible phenotypes are those that we expect to find in nature as outcomes of past evolutionary processes. Two objections have been raised against this premise, and both are based on the observation that adaptive evolution can usually proceed only gradually by means of mutations of small phenotypic effect.

The critical question is whether a strategy identified as evolutionarily stable is actually attainable by small mutational steps from at least some ancestral states. A first issue, recognized early on in the modern synthesis and leading to Wright's shifting-balance theory (Wright 1931, 1932, 1967, 1988), is that global fitness maxima may often not be attainable, since the evolutionary process becomes stuck on a local fitness maximum. This lends weight to the notion of a "local ESS", already highlighted above. A second, and completely separate, issue arises from the presence of frequency dependence, under which evolutionary stability and attainability turn out to part company (Eshel and Motro 1981; Eshel 1983). This means that gradual evolution may lead away from ESSs, and that, even more disturbingly, outcomes actually attained by gradual evolution may not be ESSs. Only within the restricted realm of optimization approaches is this second problem absent (Meszéna et al. 2001; Box 11.2). 
Box 11.1 Limitations of optimization in life-history evolution

Here we illustrate the critical consequences of environmental feedback, using the evolution of age at maturation as an example. By referring to models developed by Mylius and Diekmann (1995) and by Heino et al. (1997b) we make two important points: (1) when environmental feedback is one-dimensional and monotonic, evolution is optimizing - but even so only rarely can it be reduced to the maximization of $r$ or $R_{0}$; and (2) optimization approaches lose their validity whenever the environmental feedback is more than one-dimensional.

Environmental feedback refers to the full description of the environment as it occurs in the feedback loop in the considered population dynamics. In general, for populations that attain stable equilibria, the dimension of the feedback environment is the minimal number of variables that, independently of the mutant trait value, are sufficient to characterize the environment established by a resident population for the dynamics of a rare mutant population (Metz et al. 1996b).

One-dimensional environmental feedback. We consider an organism's life history as follows (Mylius and Diekmann 1995). Juveniles mature into adults at age $x$, after which they produce offspring at a constant rate $b$. Juveniles and adults die at rates $d_{\mathrm{J}}$ and $d_{\mathrm{A}}$, respectively. All of these parameters can be affected by the environment $E$, as a consequence of the feedback loop. We denote their values in the virgin environment $E_{\mathrm{V}}$ (the environment unaffected by the population) by the subscript $\mathrm{V}$. The adaptive trait considered here is $x_{\mathrm{V}}$. Postponed maturation leads to an increased adult reproductive rate, $b\left(x_{\mathrm{V}}\right)=\max \left(0, x_{\mathrm{V}}-1\right)$. This means that $b$ is 0 for $x_{\mathrm{V}}<1$ and that it equals $x_{\mathrm{V}}-1$ otherwise. Three alternative feedback loops are investigated: (1) $E$ only affects juvenile and adult mortality rates by an equal additional term for both; (2) $E$ only affects juvenile mortality rate additively; and (3) $E$ only affects the age at maturation multiplicatively. For each feedback scenario, parameters not affected by the environment take on their value in the virgin environment. For fixed values of $x_{\mathrm{V}}$ and $E$, the basic reproductive ratio $R_{0}\left(x_{\mathrm{V}}, E\right)$ is given by

$$
R_{0}\left(x_{\mathrm{V}}, E\right)=\frac{b\left(x_{\mathrm{V}}\right)}{d_{\mathrm{A}}(E)} e^{-d_{\mathrm{J}}(E) x\left(x_{\mathrm{V}}, E\right)} .
$$

Also, the population's intrinsic rate of increase $r\left(x_{\mathrm{V}}, E\right)$ can be obtained as the unique real root of the corresponding Euler-Lotka equation (e.g., Roughgarden 1979; Yodzis 1989),

$$
\frac{b\left(x_{\mathrm{V}}\right) e^{-\left[r\left(x_{\mathrm{V}}, E\right)+d_{\mathrm{J}}(E)\right] x\left(x_{\mathrm{V}}, E\right)}}{r\left(x_{\mathrm{V}}, E\right)+d_{\mathrm{A}}(E)}=1 .
$$

It turns out that only for feedback scenario (1) does adaptive evolution maximize $r$. Consequently, one can determine the evolutionary optimum $x_{\mathrm{V}}^{*}$ by maximizing $r\left(x_{\mathrm{V}}, E\right)$ with respect to $x_{\mathrm{V}}$, either for $E=E_{\mathrm{V}}$ or for any other fixed $E$. For feedback scenario (2), the quantity maximized by evolution turns out to be $\left[\ln R_{0}\left(x_{\mathrm{V}}, E_{\mathrm{V}}\right)\right] / x_{\mathrm{V}}$. This is not equivalent to maximizing $R_{0}\left(x_{\mathrm{V}}, E_{\mathrm{V}}\right)$. Instead, the optimized quantity can be rewritten as $\left[\ln b\left(x_{\mathrm{V}}\right)\right] / x_{\mathrm{V}}$, which is also the quantity that is evolutionarily maximized for feedback scenario (3).

This first example thus highlights that the appropriate fitness measure maximized by evolution under a one-dimensional environmental feedback loop clearly depends on the mode of density dependence, and only under special conditions reduces to $r$ or $R_{0}$.

continued 


\section{Box 11.1 continued}

Two-dimensional environmental feedback. A multidimensional feedback environment can only occur when there is some structure in the considered population. This structure can be genetic, social, temporal, spatial, or physiological (i.e., age-, stage-, or size-structured) and enables different individuals to have a different influence on, as well as a different perception of, the environment. Thus, whether or not a particular population structure creates a multidimensional feedback environment depends on how these aspects of influence and perception are specified in the considered population dynamics model.

As a typical example, the following model-simplified from Heino et al. (1997b) - investigates a population structured in two age classes. The species is semelparous, and individual transitions between classes take one time unit (e.g., 1 year). Maturity can be reached within the first year of life, or delayed until the second year. The adaptive trait is the probability of maturing at age 1 , denoted by $x$. The other life-history parameters - intrinsic age-specific survival $s_{i}(i$ refers to ages 0 and 1 ) and intrinsic fecundity $b_{i}$ (with $i=1,2$ ) - are potentially affected during any year $t$ by a two-dimensional environment $\left\{E_{1}(t), E_{2}(t)\right\}$. Transitions between age classes are as follows. Recruitment into age 1 from age 1 and 2: the per capita number of recruited individuals at time $t+1$ is given by $s_{0} b_{1} x /\left[1+c_{1} E_{1}(t)\right]$ and $s_{0} b_{2} /\left[1+c_{1} E_{1}(t)\right]$, respectively, where $c_{1}$ is a scaling parameter. Survival from age 1 to age 2: the survival probability is given by $s_{1}(1-x) /\left[1+c_{2} E_{2}(t)\right]$, where $c_{2}$ is a scaling parameter. If the population dynamics reach equilibrium, we denote the equilibrium sizes of age class 1 and age class 2 by $N_{1}^{*}$ and $N_{2}^{*}$, respectively. Recruitment is assumed to decrease with the density of newborns, and survival at age 1 decreases with the density of non-reproducing adults. The considered environmental feedback $\left\{E_{1}, E_{2}\right\}=\left\{b_{1} x N_{1}^{*}+b_{2} N_{2}^{*},(1-x) N_{1}^{*}\right\}$ is thus two-dimensional.

The evolutionarily stable fraction $x^{*}$ of individuals that mature at age 1 depends on the order of three quantities: $s_{1} b_{2}-b_{1},\left(s_{0} b_{1}-1\right) c_{2} / c_{1}$, and 0 . All individuals are predicted to mature at age 2 (age 1) if $s_{1} b_{2}-b_{1} \geq\left(s_{0} b_{1}-1\right) c_{2} / c_{1}\left(s_{1} b_{2}-b_{1} \leq 0\right)$. However, when both of these conditions are not satisfied, $0<s_{1} b_{2}-b_{1}<$ $\left(s_{0} b_{1}-1\right) c_{2} / c_{1}$, a stable polymorphism arises with $x^{*}=c_{1}\left(s_{1} b_{2}-b_{1}\right) /\left[c_{2}\left(s_{0} b_{1}-1\right)\right]$ : a fraction $0<x^{*}<1$ of individuals mature at age 1 and the remaining fraction $1-x^{*}$ at age 2 . Thus, when the dimension of the environmental feedback is greater than one, a stable phenotypic polymorphism in the age at maturity can evolve. Intuitively, this is possible because under density dependence fitness ought to vary with population density, and thus require one environmental variable; the addition of a second environmental variable makes it possible for fitness to depend also on the relative frequencies of trait values in the population. A twodimensional feedback environment is, indeed, a necessary condition (although not a sufficient one) for the evolution of stable polymorphisms. Importantly, no optimization principle can be devised to predict the evolutionarily stable fraction $x^{*}$ (Metz et al. 1996b).

The dimension of feedback environments is only sharply defined in the world of models. In reality, this dimensionality is often relatively large or even infinite, with the environmental variables involved decreasing in their importance and impact. This implies, in particular, that one-dimensional feedback environments are not actually expected to occur in nature - which means, in turn, that evolutionary optimization will almost never apply to natural systems. 
Box 11.2 Pairwise invasibility plots

Pairwise invasibility plots provide a handy way to analyze which mutant can invade which resident populations (Matsuda 1985; Van Tienderen and de Jong 1986; Metz et al. 1992, 1996a; Kisdi and Meszéna 1993; Geritz et al. 1997; see also Taylor 1989). Pairwise invasibility plots portray the sign structure of the invasion fitness $f$ across all possible combinations of one-dimensional mutant trait values $x^{\prime}$ and resident trait values $x$. Zero contour lines at which $f\left(x^{\prime}, x\right)=0$ separate regions of potential invasion success $(f>0)$ from those of invasion failure $(f<0)$. An example is shown below (left panel).

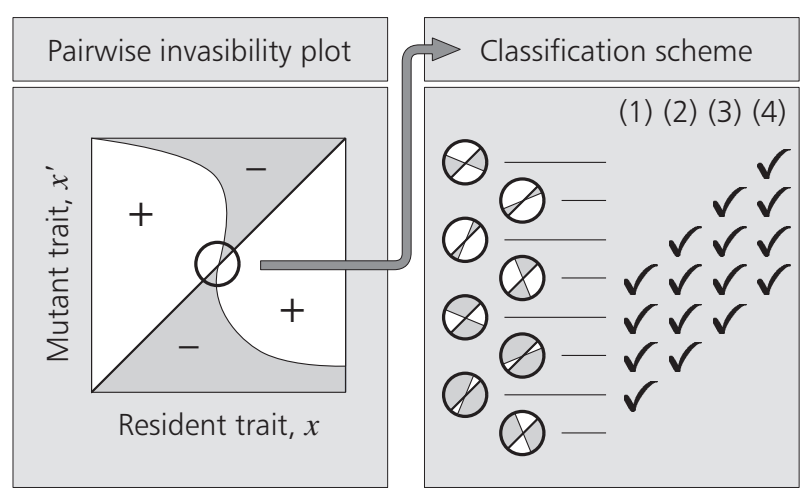

The resident trait value is neutral in its own environment, so one necessarily has $f(x, x)=0$, and the set of zero contour lines therefore always includes the main diagonal. The shape of the other zero contour lines carries important information about the evolutionary process. In particular, intersections of zero contour lines with the main diagonal define the evolutionary singularities that are possible evolutionary end-points. Evolutionary singularities can be characterized according to four properties (Geritz et al. 1997):

1. evolutionary stability;

2. convergence stability;

3. invasion potential; and

4. mutual invasibility.

Whether each of these properties applies to a given evolutionary singularity can be decided simply by looking at the pairwise invasibility plot and reading the slope of the zero contour line at the singularity, as illustrated in the right panel above.

Four interesting types of evolutionary singularities are highlighted below. In each case, the staircase-shaped line indicates a possible adaptive sequence by which evolutionarily advantageous mutants repeatedly invade and replace residents.
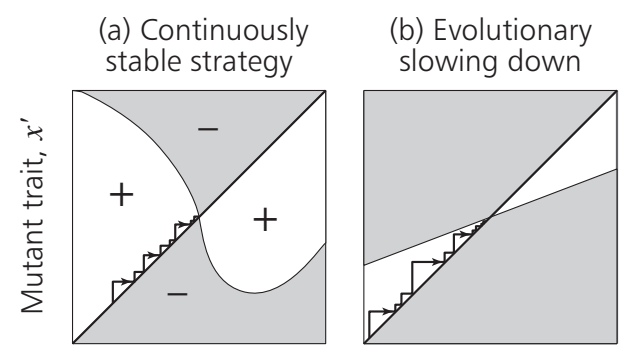

(c) Garden-of-Eden configuration

(d) Evolutionary

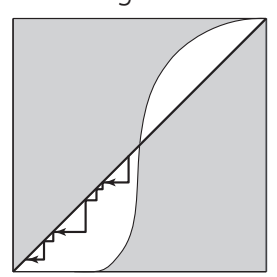
branching point

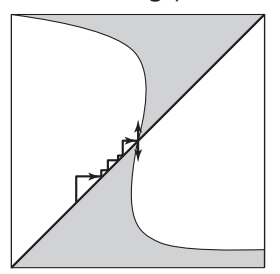

Resident trait, $x$ 


\section{Box 11.2 continued}

Panel (a) above shows a situation in which the singularity is a so-called continuously stable strategy (CSS; Eshel and Motro 1981; Eshel 1983). A CSS is both evolutionarily stable and convergence stable, and thus serves as a likely endpoint of gradual evolutionary change. Panel (b) depicts a CSS that lacks invasion potential, which causes the evolutionary process to slow down algebraically as the population moves closer to the CSS (Dieckmann and Law 1996). Panel (c) illustrates a Garden-of-Eden configuration (Nowak and Sigmund 1989), an ESS that is not convergence stable and hence cannot be attained by small mutational steps. Panel (d) shows an evolutionary branching point (Metz et al. 1992, 1996a), in which the singularity is convergence stable, but not evolutionarily stable, and nearby mutants are mutually invasible. Such configurations cause disruptive selection and thus permit the phenotypic divergence of two subpopulations that straddle the branching point.

\section{Optimization and population viability}

Even when restricting attention to those models that allow evolutionary outcomes to be predicted through $r$ or $R_{0}$ maximization, the assumption that population viability would be maximized as well is incorrect. This can be shown easily with a simple example.

For this purpose we consider a population of organisms with nonoverlapping generations regulated by Ricker-type density dependence (Chapter 2). A life-history trait $x$ influences the population's intrinsic growth rate $r$ such that its dynamics are governed by the recursion equation $N_{t+1}(x)=$ $r(x) \exp \left(-\alpha N_{t}(x)\right) N_{t}(x)$, where $N_{t}$ denotes the population size at time $t$ and $\alpha$ measures the strength of density dependence. A mutant trait value $x^{\prime}$ can invade a resident population of $x$ individuals if the mutant population's geometric growth rate in the environment set by the resident exceeds 1 , that is, if $\left[\prod_{t=0}^{T-1} r\left(x^{\prime}\right) \exp \left(-\alpha N_{t}(x)\right)\right]^{1 / T}>1$ for large durations $T$. The resident population is at ecological equilibrium if $\left[\prod_{t=0}^{T-1} r(x) \exp \left(-\alpha N_{t}(x)\right)\right]^{1 / T}=1$ for large durations $T$, which, together with the previous inequality, yields the simple invasion criterion $r\left(x^{\prime}\right)>r(x)$. Thus, evolution in this model is expected to maximize $r$ as a function of the trait $x$. The existence of such an optimization principle is the consequence of a one-dimensional eco-evolutionary feedback: all individuals perceive the same environment, characterized by the size of the whole population. It is readily shown that the average asymptotic population size of an $x$-population is $(1 / \alpha) \ln r(x)$, which implies that this population size is evolutionarily maximized together with $r$. The same conclusion, however, does not extend to population viability: as $r$ increases in the course of evolution, the population equilibrium becomes unstable and is replaced with oscillations (cycles or chaos) of increasing amplitude, with the lowest population size approaching zero (May and Oster 1976; Gatto 1993), thus increasing the risk of extinction through demographic stochasticity (Allen et al. 1993; Renault and Ferrière, unpublished). We must therefore conclude that, although evolution in this example follows an optimization principle, it nevertheless drives up the risk of population extinction. 
This section shows that the conventional approach of maximizing $r$ or $R_{0}$ to study life-history evolution is fraught with fundamental limitations. In the next section we introduce the theory of adaptive dynamics as an extended framework that overcomes these limitations, while it encompasses the classic theory as a special case.

\subsection{Adaptive Dynamics Theory}

Whenever an ecological system adapts, it affects its environment, which in turn can modify the selective pressures that act on the system: as the preceding section shows, the resultant eco-evolutionary feedback is critical for describing adaptive evolution.

\section{Invasion fitness}

The fitness of organisms can only be evaluated relative to the environment in which they live. Eco-evolutionary feedback means that this environment depends on the current adaptive state of the population under consideration. To assess the fitness of a variant phenotype, one must therefore specify the resident phenotype against which the variant is competing. In adaptive dynamics theory this is accomplished by the concept of invasion fitness (Metz et al. 1992). This quantity measures the long-term per capita growth rate $f$ of a phenotype $x$ in a given environment $E, f=$ $f(x, E)$. The environment $E$ is determined by externally fixed parameters and by the population density and phenotype of the resident population(s). Thus, the invasion fitness of a variant readily accounts for the consequences of frequencydependent ecological interactions. If the variant has an advantage compared with the resident - that is, if it has positive invasion fitness - it can spread through the population; by contrast, if the variant has negative invasion fitness, it will quickly become extinct.

Remarkably, the analysis of invasion fitness provides important insights into the dynamics and outcome of adaptive evolution, as long as it is justified to assume that the environment $E$ has settled to a stationary state determined by the resident set of phenotypes. Under that assumption, we can replace the dependence of invasion fitness on the current environment $E$ with a dependence on the resident phenotypes $x_{1}, x_{2}, \ldots, f=f\left(x, x_{1}, x_{2}, \ldots\right)$. In general, these phenotypes can belong to the same species as the variant phenotype $x$ does, or they can involve other, coevolving species (see Chapters 16 and 17 for applications of the adaptive dynamics framework in the context of coevolution). If the community of resident phenotypes possesses coexisting attractors, invasion fitness is usually multi-valued, as the environmental conditions engendered by the resident phenotypes then depends on which attractor is attained. For the sake of simplicity, it is often sufficient to characterize a population by its prevalent or average phenotype (Abrams et al. 1993). Although strictly monomorphic populations are seldom found in nature, it turns out that the dynamics of polymorphic populations (harboring, at the same time, many similar phenotypes per species) can often be well described and understood in terms of the simpler monomorphic cases. 


\section{Evolutionary singularities and their properties}

For a single species we can thus consider the invasion fitness $f=f\left(x^{\prime}, x\right)$ of a variant phenotype $x^{\prime}$ in a resident population of phenotype $x$. The sign structure of these functions can be depicted graphically to produce so-called pairwise invasibility plots, which carry important information about the evolutionary process (Box 11.2).

In particular, pairwise invasibility plots clearly identify potential evolutionary endpoints at which selection pressures vanish. These potential endpoints are called evolutionary singularities and are characterized by the following four properties:

- Evolutionary stability. Is a singularity immune to invasion by neighboring phenotypes? This property defines a local version of the classic ESS that lies at the heart of evolutionary game theory (Hamilton 1967; Maynard Smith and Price 1973; Maynard Smith 1982).

- Convergence stability. When starting from neighboring phenotypes, do successful invaders lie closer to the singularity? Here the attainability of the singularity is under consideration, an issue separate from its invasibility (Eshel and Motro 1981; Eshel 1983).

- Invasion potential. Is the singularity able to invade populations of neighboring phenotypes (Kisdi and Meszéna 1993)?

- Mutual invasibility. If a pair of neighboring phenotypes lie on either side of a singularity, can they invade into each other? Assessment of this possibility is essential to predict coexisting phenotypes and the emergence of polymorphisms (Van Tienderen and de Jong 1986; Metz et al. 1992, 1996a).

Among the eight feasible combinations of these properties (Metz et al. 1996a; Geritz et al. 1997), some have striking implications for the adaptive process:

- Convergence and evolutionary stability. The first two properties in the list above characterize a so-called continuously stable strategy (CSS; Eshel 1983). Processes of gradual adaptation experience a considerable slowing down when they converge toward a CSS (Dieckmann and Law 1996); this deceleration is most pronounced in the absence of invasion potential.

- Evolutionary stability without convergence stability. Although the singularity is resistant against invasion from all nearby phenotypes, it cannot be attained by small mutational steps - a situation aptly referred to as a Garden-of-Eden configuration by Nowak and Sigmund (1989). The existence of this type of evolutionary singularity echoes one of the limitations of optimization approaches highlighted in the previous section.

- Convergence stability without evolutionary stability. Convergence stability does not entail that the singularity be evolutionarily stable. In the absence of evolutionary stability, selection becomes disruptive near a convergence-stable singularity. Two phenotypically distinct subpopulations can then diverge from around the singularity in a process called evolutionary branching (Metz et al. 1992, 1996a; Geritz et al. 1997). 
Box 11.3 Models of adaptive dynamics

The theory of adaptive dynamics derives from consideration of ecological interactions and phenotypic variation at the level of individuals. Extending classic birth and death processes, adaptive dynamics models keep track, across time, of the phenotypic composition of a population in which offspring phenotypes are allowed to differ from those of their parents.

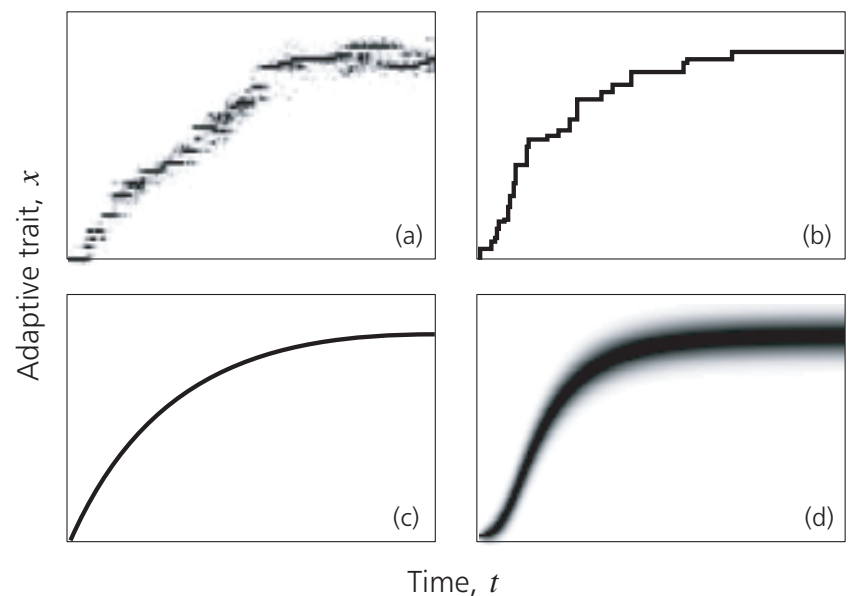

Four types of models are used to investigate adaptive dynamics at different levels of resolution and generality:

- At any time the population can be represented in trait space as a cloud of points, each point corresponding to an individual's combination of trait values. This polymorphic cloud of points stochastically drifts and diffuses as a result of selection and mutation (Dieckmann 1994; Dieckmann et al. 1995), see panel (a).

- In large populations characterized by a low mutation rate, evolutionary change in clonal species proceeds through sequences of trait substitutions (Metz et al. 1992). During each such step, a mutant with positive invasion fitness quickly invades a resident population, ousting the former resident. These steps can be analyzed through the pairwise invasibility plots introduced in Box 11.2. Concatenation of such substitutions produces a directed random walk of the type depicted in panel (b) above. Formally, such random-walk models are obtained from the process in panel (a) by considering the case of rare mutations (Dieckmann 1994; Dieckmann et al. 1995; Dieckmann and Law 1996).

- If, in addition, mutation steps are sufficiently small, the staircase-like dynamics of trait substitutions are well approximated by smooth trajectories, see panel (c) above. These trajectories follow the canonical equation of adaptive dynamics (Dieckmann 1994; Dieckmann et al. 1995; Dieckmann and Law 1996), which in its simplest form is

$$
\frac{d x}{d t}=\left.\frac{1}{2} \mu \sigma^{2} N^{*}(x) \frac{\partial f\left(x^{\prime}, x\right)}{\partial x^{\prime}}\right|_{x^{\prime}=x},
$$

where $x$ is the adaptive trait, $\mu$ is the probability for mutant offspring, $\sigma^{2}$ is the variance of mutational steps, $N^{*}(x)$ is the equilibrium size of a population with resident trait value $x$, and $f$ is the invasion fitness. The partial derivative in the equation above is the selection gradient $g(x)$. Evolutionary singularities are trait values $x^{*}$ for which the selection gradient vanishes, $g\left(x^{*}\right)=0$. 


\section{Box 11.3 continued}

In large populations characterized by high mutation rates, stochastic elements in the dynamics of the phenotypic distributions become negligible; this enables mathematical descriptions of reaction-diffusion type (Kimura 1965; Bürger and Bomze 1996; Bürger 1998), see panel (d) above. However, the infinitely extended tails that phenotypic distributions instantaneously acquire in this framework often give rise to artifactual dynamics that have no correspondence to processes that could occur in any finite population (Mollison 1991; Cruickshank et al. 1999).

At the expense of ignoring genetic complexity, models of adaptive dynamics are geared to analyze the evolutionary implications of ecological settings. This allows all types of density- and frequency-dependent selection mechanisms to be studied within a single framework, into which coevolutionary dynamics driven by interspecific interactions are also readily incorporated (Dieckmann and Law 1996; Chapters 16 and 17). Extensions are also available to describe the evolution of multivariate traits (Dieckmann and Law 1996) and of function-valued traits (Dieckmann and Heino, unpublished).

As long as the adaptive process stays away from evolutionary branching points, the evolutionary dynamics follow selection gradients determined by the first derivative of invasion fitness in the direction of the variant trait, and can be described by a simple differential equation known as the canonical equation of adaptive dynamics (Box 11.3).

In the next two sections we utilize adaptive dynamics theory to investigate two remarkable consequences of closing the eco-evolutionary feedback loop:

- Natural selection can play a major role in driving the diversification of communities.

- Natural selection can cause population extinction, even in the absence of environmental change.

\subsection{Adaptive Evolution and the Origin of Diversity}

The response of biodiversity to environmental changes is likely to span a continuum, from the immediate ecological consequences to longer-term evolutionary effects. Both ends of this continuum raise conservation concerns.

\section{Conservation and speciation}

On the ecological time scale, global biodiversity can only be lost. Locally, of course, biodiversity may be enhanced by the invasion of exotic species, but even that often leads to the subsequent loss of native species (Drake et al. 1989; Williamson 1996; Mooney and Hobbs 2000; Mooney and Cleland 2001; Perrings et al. 2002). By contrast, on the evolutionary time scale, not only can biodiversity 
be lost (Section 11.5), but also it can be generated, which thus has conservation implications: "Death is one thing, an end to birth is something else", in the words of Soule (1980). The "birth" process in ecological communities is speciation, for which human activities are suggested to have at least three major repercussions (Myers and Knoll 2001):

- Outbursts of speciation. As large numbers of niches are vacated, there could be explosive adaptive radiations within certain taxa - notably small mammals, insects, and terrestrial plants - able to thrive in human-dominated landscapes.

- Reduced speciation rates. Biogeography theory suggests that speciation rates correlate with area (e.g., Rosenzweig 1995, 2001; Losos 1996; Losos and Schluter 2000). Therefore even the largest protected areas and nature reserves may prove far too small to support the speciation of large vertebrates. Even for smaller species, habitat fragmentation may severely curb speciation rates.

- Depletion of evolutionary powerhouses. The unrelenting depletion and destruction of tropical biomes that have served in the past as pre-eminent powerhouses of evolution and speciation (Jablonski 1993) could entail grave consequences for the long-term recovery of the biosphere.

The long-term, macro-evolutionary character of hypotheses like those above means they are notoriously difficult to evaluate empirically. Models that do justice to the underlying mechanisms have to be reasonably complex, which appears to deter theorists from tackling these questions. However, at least the first two notions in the list above have received some attention from modeling and theory. Below we summarize recent studies that bear on these issues.

\section{Determinants of evolving biodiversity}

Law (1979) introduced the "Darwinian Demon" as a hypothetical organism that has solved all challenges of life-history evolution - it starts to reproduce immediately after birth, produces very large numbers of offspring at frequent intervals, supplies each offspring with massive food reserves that ensure survival, possesses a high longevity, disperses well, finds mates at will, and it can achieve all these successes in any habitat. Evidently, such a super-organism would quickly take over the earth's biosphere and would thus eradicate all diversity. This illustrates that understanding biodiversity always entails understanding the life-history trade-offs that prevent such demons from arising: ecological coexistence is possible because of such trade-offs. In this vein, many biodiversity models (e.g., Hastings 1980; Tilman 1994; Tilman et al. 1994; May and Nowak 1994; Nowak and May 1994) focused on species assemblages that are ecologically stable. Yet most ecologically stable communities are not evolutionarily stable. To describe processes that go beyond short-term responses to environmental change, we must learn to understand the mechanisms and environmental determinants that generate and maintain diversity in evolving communities. The two models described next address this question by analyzing, respectively, evolution under trade-offs between competition and dispersal, and between growth and fecundity. 
Modeling the exposure of a formerly nitrogen-poor community of terrestrial plants to a large increase in the rate of nitrogen deposition, Tilman and Lehman (2001) considered the community's response both at the ecological and the evolutionary time scale. Unsurprisingly, their model predicts that the short-term effect of the environmental change is the take-over of a few formerly rare but now fastgrowing and rapidly dispersing species. The differential success of these plants is enhanced by asymmetric competition for light. After the initial ecological response, evolutionary processes come into play and reshape the entire community. Based on a trade-off between competitive ability and dispersal potential, the model predicts that the winners of the short-term round gradually reduce their capacity to disperse by evolving into progressively better local competitors. To justify their reaction-diffusion modeling of adaptive dynamics (see Box 11.3), Tilman and Lehman (2001) assumed that mutations are so frequent that, at any time, the community always features a wide range of phenotypes at low density. Under such conditions, evolution first establishes two distinct morphs: a good disperser that is a poor competitor and a good competitor that is a poor disperser. Afterwards, the former morph again evolves toward better competitive ability and thus allows a well dispersing third morph to invade with traits similar to those the first and second morph had both possessed initially. Thus, the range between the two extreme strategies successively fills with a collection of intermediate species. Tilman and Lehman (2001) describe this pattern as the result of a speciation process that eventually yields a local flora that is as species rich as that before the environmental change. The far-reaching conclusion from this theoretical study is that rapid speciation processes can confer high long-term resilience to the diversity of natural communities against the immediate negative impacts of habitat degradation.

A different model of biodiversity evolution was analyzed by Jansen and Mulder (1999; see also Mouquet et al. 2001) to describe a seasonal community of selfpollinating plants that inhabited a large collection of patches. Throughout the season, competing plant species grow within patches of equal carrying capacity. At the end of the season, the plant biomass thus accrued is converted back into seeds, which are then distributed randomly across all patches. Plant species differ in a single quantitative trait that describes their growth rate; fecundity is negatively correlated with growth and vanishes at a given maximal growth rate, while competitive ability and dispersal potential are independent of the trait. Evolution is enabled by a small probability that a seed is a mutant, in which case its growth rate slightly differs from its parent. Figure 11.1 shows how biodiversity in the evolved species assemblages depends on season length and environmental quality:

- Predicted biodiversity increases with season length. This is because longer seasons select for fast-growing but less fecund phenotypes, which results in a larger fraction of patches being unoccupied by fast-growing phenotypes and thus open to more slowly growing phenotypes. The finding is compatible with observed biodiversity, which increases toward the equator. 


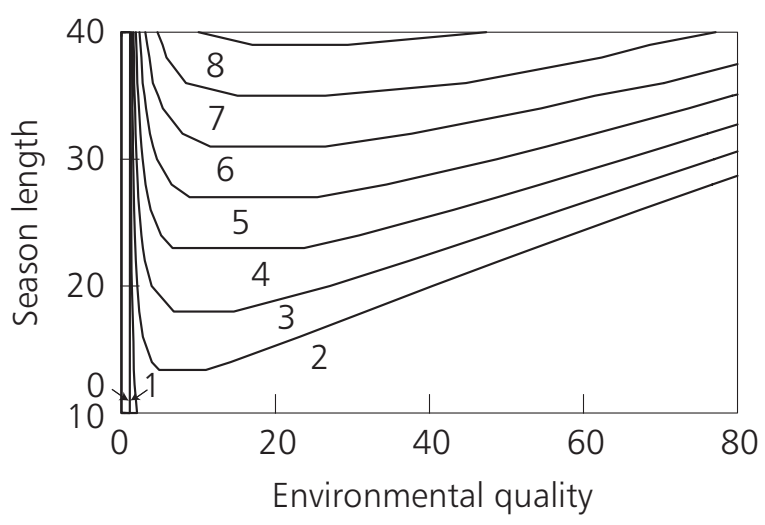

Figure 11.1 Patterns of biodiversity that emerge from the adaptive dynamics of a competitive plant community. Predicted biodiversity, measured as the number of species in the evolutionarily stable community, changes at the contour lines, increasing with season length and exhibiting a maximum for local environments of intermediate quality. Source: Jansen and Mulder (1999).

- Predicted biodiversity is maximal for environments of intermediate quality. Rich environments, here defined as featuring patches of high carrying capacity, lead to high total fecundity and thus to a saturated situation in which most patches are occupied by the types that grow fastest, which drives any other types to extinction. By contrast, poor environments lead to low total fecundity and thus to a situation in which diversity is "starved" by the rare colonization of patches. These antagonistic effects cause the model's biodiversity to peak at a medium environmental quality. Also this prediction is in accordance with observed productivity-diversity relations (Rosenzweig 1995).

We may thus expect diversity patterns to follow environmental conditions predictably, as these change over space or time. Once corroborated and complemented by more detailed ecological models, such insights may help to diagnose community-level disturbances caused by environmental change, and, where necessary, to devise recovery measures that restore the evolutionary potential and/or stability of affected species assemblages.

\section{Adaptive speciation}

The two models discussed above are based on a phenotypic representation of quantitative traits. Their utility lies in highlighting the ecological and environmental conditions conducive to adaptive radiation and necessary to maintain diverse communities. A critical element in both models is frequency-dependent selection, which allows, as shown in Section 11.3, evolving populations to converge through directional selection to fitness minima, at which selection turns disruptive. The key point to appreciate here is that under such circumstances, which cannot arise in models of life-history optimization, the splitting of a lineage trapped at a fitness minimum becomes adaptive. The resultant processes of adaptive speciation (Dieckmann et al. 2004) are very different from those stipulated by the standard 
model of allopatric speciation through geographic isolation, which dominated speciation research for decades (Mayr 1963, 1982). Closely related to adaptive speciation are models of sympatric speciation (e.g., Maynard Smith 1966; Johnson et al. 1996), of competitive speciation (Rosenzweig 1978), and of ecological speciation (Schluter 2000), which all indicate the same conclusion: patterns of species diversity are not only shaped by processes of geographic isolation and immigration, which can be more or less random, but also by processes of selection and evolution, which are bound to infuse such patterns with a stronger deterministic component.

When considering adaptive speciation in sexual populations, selection for reproductive isolation comes into play. Since at evolutionary branching points lineage splits are adaptive, in the sense that populations are freed from being stuck at fitness minima, premating isolation is expected to evolve more readily under such circumstances than previously believed. Any evolutionarily attainable or already existing mechanism of assortative mating can be recruited by selection to overcome the forces of recombination that otherwise prevent sexual populations from splitting up (e.g., Felsenstein 1981). Since a plethora of such mechanisms exist for assortativeness (based on size, color, pattern, acoustic signals, mating behavior, mating grounds, mating season, the morphology of genital organs, etc.), and since only one of these many mechanisms needs to take effect, it would be surprising if many natural populations remained stuck at fitness minima for very long (Geritz et al. 2004). Models for the evolutionary branching of sexual populations corroborate this expectation (Dieckmann and Doebeli 1999, 2004; Doebeli and Dieckmann 2000; Geritz and Kisdi 2000; Box 11.4).

In conjunction with mounting empirical evidence that rates of race formation and sympatric speciation are potentially quite high, at least under certain conditions (e.g., Bush 1969; Meyer 1993; Schliewen et al. 1994), the above considerations suggest that longer-term conservation efforts will benefit if attention is paid to how environmental change interferes with evolutionarily stable community patterns.

\section{Area effects on adaptive speciation}

Doebeli and Dieckmann $(2003,2004)$ incorporated spatial structure into models of evolutionary branching. They found that, even in the absence of any significant isolation by distance, spatial environmental gradients could greatly facilitate adaptive parapatric speciation. Such facilitation turned out to be most pronounced along spatial gradients of intermediate slope, and to result in stepped biogeographic patterns of species abutment, even along smoothly varying gradients. These findings are explained by observing that the combination of local adaptation and local competition along a gradient acts as a potent source of frequency-dependent selection. The investigated models allow substantial gene flow along the environmental gradient, so isolation by distance does not offer an alternative explanation for the observed phenomena. 
Box 11.4 Sympatric speciation in sexual populations

Sympatric speciation in sexual populations necessarily involves a sufficiently high degree of reproductive isolation - otherwise hybrids occupy any potentially developing gap between the incipient species. Apart from chromosomal speciation, which involves incompatible levels of ploidy, reproductive isolation in sympatry is most likely to occur through a prezygotic mechanism that results in assortative mating. Unless assortativeness is already present for some reason, it thus has to evolve in the course of sympatric speciation.

Dieckmann and Doebeli (1999) considered a simple model with two adaptive traits: first, an ecological character exposed to selection pressures that would lead to evolutionary branching in an asexual population, and second, a variable degree of assortativeness on the ecological character. Both traits were modeled with diploid genetics, assuming sets of equivalent diallelic loci with additive effects and free recombination. Under these conditions, sympatric speciation happens easily and rapidly. This is illustrated by the sequence of panels below, in which both quantitative traits are coded for by five loci, thus giving rise to a quasi-continuum of 11 different phenotypes. In each panel, gray scales indicate the current frequencies of the resultant $11^{2}=121$ phenotypic combinations in the evolving population (the highest frequency in a panel is shown in black, with a linear transition of gray scales to frequency zero, shown in white).

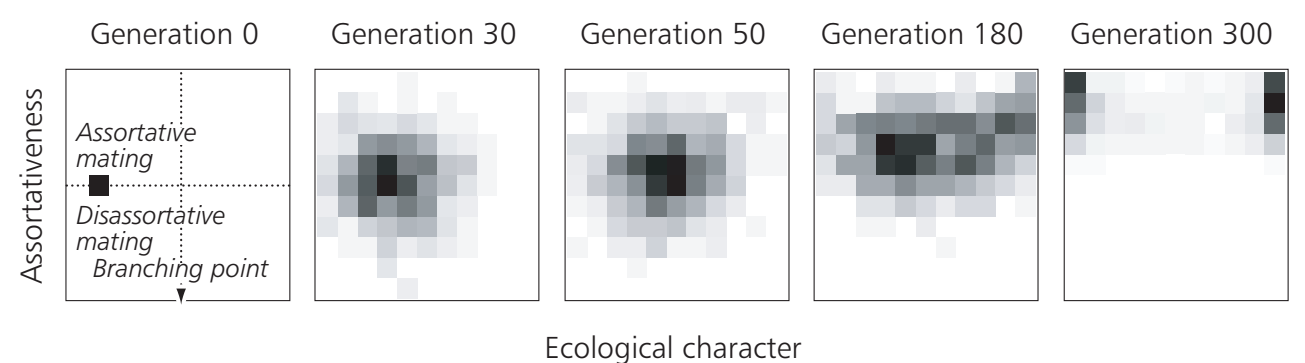

The above sequence of events starts out with random mating, away from the evolutionary branching point. After the population has converged to the branching point, it still cannot undergo speciation, since recombination under random mating prevents the ecological character from becoming bimodal. However, if the disruptive selection at the branching point is not too weak (Matessi et al. 2001), it selects for increased assortative mating. Once assortativeness has become strong enough, speciation can occur. Eventually, the ecological characters of the incipient species diverge so far, and assortive mating becomes so strong, that hardly any hybrids are produced and the gene flow between the two species essentially ceases.

In a second, related model, Dieckmann and Doebeli (1999) considered an additional quantitative character that is ecologically neutral and only serves as a signal upon which assortative mating can act. Numerical analysis shows that in this case sympatric speciation also occurs. Conditions for speciation are only slightly more restrictive than in the first model, even though a linkage disequilibrium between the ecological character and the signal now has to evolve as part of the speciation process.

These results support the idea that when frequency-dependent ecological interactions cause a population to converge onto a fitness minimum, solutions can often evolve that allow the population to escape from such a detrimental state. This makes the speciation process itself adaptive, and underscores the importance of ecology in understanding speciation. 
These findings, which were obtained for models of both asexual and sexual populations, could have repercussions in terms of understanding species-area relationships, widely observed in nature. Species diversity tends to increase with the size of the area over which diversity is sampled, a characteristic relationship that is often described by power laws (Rosenzweig 1995). It is therefore noteworthy that the speciation mechanism highlighted by Doebeli and Dieckmann (2003, 2004) also lets the emerging number of species increase with the total area covered by the environmental gradient. Of course, a shorter gradient in a smaller area often covers a reduced range of environmental heterogeneity compared with an extended gradient in a larger area. So one component of species-area relationships is expected to originate from the enhanced diversity of environmental conditions that in turn supports a greater diversity of species. Interestingly, however, Doebeli and Dieckmann (2004) found that their model predicted larger areas to harbor more species than smaller areas, even when both areas featured the same diversity of environmental conditions. This suggests that a second component of species-area relationships originates because the evolutionary mechanism of adaptive speciation operates more effectively in larger than in smaller areas.

Other mechanisms are also likely to contribute to species-area relationships. MacArthur and Wilson (1967), for example, based a classic explanation on their "equilibrium model of island biogeography". This model relies on the assumption that equilibrium population sizes increase linearly with island size, so that species extinctions occur more rarely on larger islands. Adopting a purely ecological perspective, their argument makes no reference to the effect of island area on the rate at which species are being formed, rather than being destroyed. By contrast, Losos and Schluter (2000) argued that the greater species richness of Anolis lizards found on larger islands in the Antilles is because of the higher speciation rates on larger islands, rather than higher immigration rates from the mainland or lower extinction rates. Since the diversity of environmental conditions does not appear to be significantly lower on smaller islands in the Antilles (Roughgarden 1995), and since, nevertheless, the big islands of the Greater Antilles typically harbor many species of Anolis lizards compared to the smaller islands of the Lesser Antilles (which contain at most two species), the second component of species-area relationships as described above may have played an important role for anole radiations in the Antilles.

This brief discussion again underscores that traditionally envisaged ecological factors of diversity must be complemented by additional evolutionary factors (this also applies to understanding species-area relationships). The effect of habitat loss and habitat fragmentation on speciation rates might thus become an important focus of evolutionary conservation biology.

\subsection{Adaptive Evolution and the Loss of Diversity}

The notion that optimizing selection maximizes an evolving population's viability leaves no room for (single-species) evolution that causes population extinctions. An appreciation of evolution's role in culling biodiversity therefore requires that the narrow concept of optimizing selection be overcome, as discussed in Section 11.1. 


\section{Evolutionary deterioration, collapse, and suicide}

Given the long tradition of describing evolutionary processes through concepts of progress and optimization, we must reiterate that no general principle actually prevents adaptive evolution from causing a population to deteriorate (Section 11.1). Even selection-driven population collapse and extinction are not ruled out and, in fact, these somewhat unexpected outcomes readily occur in a suite of plausible evolutionary models.

Evolutionary suicide (Ferrière 2000) is defined as a trait substitution sequence driven by mutation and selection that takes a population toward and across a boundary in the population's trait space beyond which the population cannot persist. Once the population's phenotypic traits have evolved close enough to this boundary, mutants that are viable as long as the current resident trait value abounds, but that are not viable on their own, can invade. When these mutants start to invade the resident population they initially grow in number. However, once they have become sufficiently abundant, concomitantly reducing the former resident's density, the mutants bring about their own extinction. This is not unlike the "Trojan gene effect" discussed by Muir and Howard (1999), although the latter does not involve gradual evolutionary change.

Two other adaptive processes are less drastic than evolutionary suicide. First, adaptation may cause population size to decline gradually in a process of perpetual selection-driven deterioration. Sooner or later, demographic and/or environmental stochasticity then cause population extinction. Second, the population collapse brought about by an invading mutant phenotype may not lead to population extinction, but only to a substantial reduction in population size. Such a collapse renders the population more susceptible to extinction by stochastic causes.

The three phenomena of population deterioration, collapse, and suicide have often been discussed in the context of evolving phenotypic traits related to competitive performance. A verbal and lucid example of evolutionary deterioration comes from overtopping growth in plants. Taller trees receive more sunlight while casting shade onto their neighbors. As selection causes the average tree height to increase, fecundity declines because more of the tree's energy budget is diverted from seed production to wood production. Under these circumstances it may also take longer and longer for the trees to reach maturity. Thus, arborescent growth as an evolutionary response to selection for competitive ability can cause population abundance and/or the intrinsic rate of population growth to decline. The logical conclusion of this process may be a population's extinction, as first explained by Haldane (1932).

Below we outline the analysis of several models that provide a mathematical underpinning to Haldane's considerations and that illustrate, in turn, processes of evolutionary deterioration, evolutionary collapse, and evolutionary suicide. All three models consider a single species with population dynamics influenced by a quantitative trait that measures competitive ability (e.g., adult body size). Variation in this phenotype is assumed to result in asymmetric competition: individuals that are at a competitive advantage by attaining larger body size at the same time suffer 


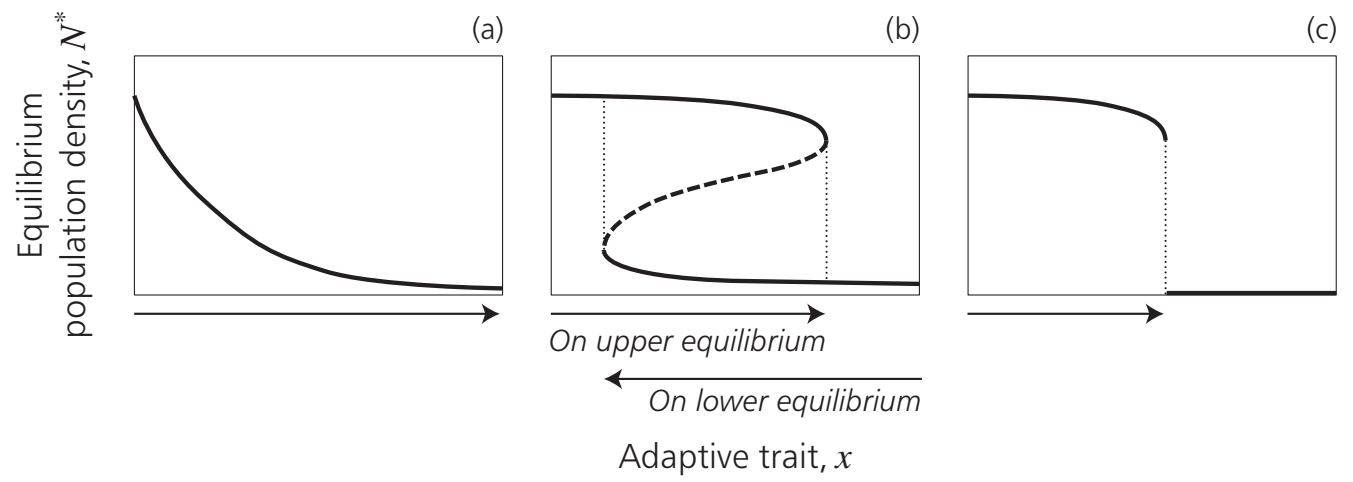

Figure 11.2 Evolutionary deterioration, collapse, and suicide. (a) Evolutionary deterioration as in the model by Matsuda and Abrams (1994a). (b) Evolutionary collapse as in the model by Dercole et al. (2002). (c) Evolutionary suicide as in the model by Gyllenberg and Parvinen (2001). In each case, continuous curves show how equilibrium population densities vary with the adaptive trait (body size), unstable equilibria are indicated by a dashed curve, and selection pressures on the adaptive traits are depicted by arrows.

from having to divert more energy to growth, which results in diminished reproduction or increased mortality. Asymmetric competition implies that in pairwise interactions the individual that is competitively superior to its opponent suffers less from the effects of competition than the inferior opponent.

\section{Evolutionary deterioration}

Matsuda and Abrams (1994a) analyzed a Lotka-Volterra model in which competing individuals experience asymmetric competition and a carrying capacity that depends on body size. In their model, the competitive impact experienced by an individual with body size $x$ in a population with mean body size $\bar{x}$ is $\alpha(x, \bar{x})=\exp \left(-c_{\alpha}(x-\bar{x})\right)$, and the carrying capacity for individuals with body size $x$ is $K(x)=K_{0} \exp \left(-c_{K}(x)\right)$. Here $c_{\alpha}$ is a nonlinear function that preserves the sign of its argument, and $c_{K}$ is a non-negative function that goes to infinity when its argument does.

Matsuda and Abrams (1994a) conclude that under these assumptions adaptive evolution continues to increase body size indefinitely - provided that the advantage of large body size (as described by $c_{\alpha}$ ) is big enough and the cost of increased body size (as described by $c_{K}$ ) is small enough. Since large body sizes result in small carrying capacities, adaptive evolution thus perpetually diminishes population size (Figure 11.2a), a phenomenon Matsuda and Abrams call "runaway evolution to self-extinction". Notice, however, that in this model population size never vanishes, but just continues to deteriorate. This means that additional stochastic factors, not considered in the studied deterministic model, are required to explain eventual extinction.

For a related model that focuses on the evolution of anti-predatory ability in a predated prey, see Matsuda and Abrams (1994b). The actual extinction through demographic stochasticity, predicted by Matsuda and Abrams (1994a), is demonstrated in an individual-based model by Mathias and Kisdi (2002). 


\section{Evolutionary collapse}

In a model by Dercole et al. (2002), the per capita growth rate in a monomorphic population with adult body size $x$ and population density $N(x)$ involves the logistic component $r(x)-\alpha_{0} N(x)$, in which the monotonically decreasing function $r(x)$ captures the negative influence of larger adult body size on per capita reproduction, and $\alpha_{0} N(x)$ measures the extra mortality caused by intraspecific competition. As in the previous model, the coefficient $\alpha_{0}$ measures the competitive impact individuals with the same phenotype have on each other. When two different phenotypes $x$ and $x^{\prime}$ interact, the competitive impact of $x$ on $x^{\prime}$ is $\alpha\left(x-x^{\prime}\right) N(x)$, where $\alpha$ increases with $x-x^{\prime}, \alpha(0)=\alpha_{0}$, and $\alpha^{\prime}(0)=-\beta$. Per capita growth is further diminished by a density-dependent term that accounts for an Allee effect. Such an effect may be caused either by reduced fecundity through a shortage of mating encounters in sparse populations, or by increased mortality because of the concentration of predation risk as density decreases (Dennis 1989; Chapter 2). Reducing the per capita growth rate by $\gamma N(x) /[1+N(x)]$ captures both variants, with $\gamma$ determining the Allee effect's strength. As described in Chapter 2, the resultant population dynamics can feature bistability: a stable positive equilibrium may coexist with a stable extinction equilibrium. Dercole et al. (2002) actually reduced the per capita growth rate by $\gamma N^{2}(x) /\left[1+N^{2}(x)\right]$ in an effort to add realism to the model by accounting for spatial heterogeneity in the chance of mating or predation risk. With this second choice, the population size can attain two stable equilibria $N^{*}(x)$, one at low and one at high density. When $x$ is low, only the high-density equilibrium exists; when $x$ is high, only the low-density equilibrium exists, while in-between the two stable equilibria coexist (Figure 11.2b).

The invasion fitness of a mutant $x^{\prime}$ in a resident population with phenotype $x$ is then given by $f\left(x^{\prime}, x\right)=r\left(x^{\prime}\right)-\alpha\left(x-x^{\prime}\right) N^{*}(x)-\gamma N^{* 2}(x) /\left[1+N^{* 2}(x)\right]$, which yields the selection gradient $g(x)=r^{\prime}(x)+\beta N^{*}(x)$, with $N^{*}(x)$ determined by $f(x, x)=0$. The selection gradient shows that two opposing selective pressures are at work: since fecundity decreases when adult body size increases, the term $r^{\prime}(x)$ is negative and thus favors smaller adult body size, whereas the term $\beta N(x)$ is positive and selects for larger body size. Ecological bistability can make the balance between these two selective forces dependent upon which equilibrium the resident population currently attains: a specific resident phenotype that occupies the high-density equilibrium gives the positive selective pressure more weight and thus favors increased adult body size $x$, whereas the same resident phenotype at the low-density equilibrium promotes the reduction of $x$. If the reproductive cost of larger body size is not too large [i.e., if $r^{\prime}(x)$ remains low], and/or if competitive asymmetry is strong [i.e., if $\beta$ is large], an ancestral population characterized by small body size and high abundance will evolve toward larger and larger adult body size - up to a point where the population's high-density equilibrium ceases to exist (Figure 11.2b). There the population abruptly collapses to its low-density equilibrium and suddenly faces a much-elevated risk of extinction because of demographic and environmental stochasticity. 


\section{Evolutionary suicide}

Also a model developed by Gyllenberg and Parvinen (2001) is based on asymmetric competition and incorporates an Allee effect. Their model is similar to the previous one, except for the following three features:

- Fecundity $b(x)$ peaks for an intermediate value of adult body size $x$;

- A trait- and density-independent mortality $d$ is considered; and

Rather than increasing mortality, the Allee effect reduces fecundity by the factor $N(x) /[1+N(x)]$.

These features are reflected in the model's invasion fitness, which is obtained as $f\left(x^{\prime}, x\right)=b\left(x^{\prime}\right) N^{*}(x) /\left[1+N^{*}(x)\right]-d-\alpha\left(x-x^{\prime}\right) N^{*}(x)$, with $N^{*}(x)$ again being determined by $f(x, x)=0$.

From this invasion fitness we can infer that the extinction equilibrium $N^{*}(x)=0$ is stable for all $x$. For intermediate values of $x$, two positive equilibria coexist, of which the high-density one is stable and the low-density one is unstable. The selection gradient $g(x)=b^{\prime}(x) N^{*}(x) /\left[1+N^{*}(x)\right]+\beta N^{*}(x)$ is positive for any $x$, provided that $\beta=-\alpha^{\prime}(0)$ is large enough (i.e., whenever competition is strongly asymmetric). It is thus clear that the adaptive dynamics of adult body size $x$ must drive the population to the upper threshold of adult body size, beyond which the two positive equilibria vanish and only the stable extinction equilibrium remains. In this model, therefore, adaptive evolution not only abruptly reduces population density (as in the previous example), but also causes the population to become extinct altogether. The resultant process of evolutionary suicide is illustrated in Figure 11.2c.

\section{Catastrophic bifurcations and evolutionary suicide}

It is not accidental that the two previous examples both involved discontinuous transitions in population density at the trait values where, respectively, evolutionary collapse and evolutionary suicide occurred. In fact, Gyllenberg et al. (2002) have shown (in the context of a particular model of metapopulation evolution) that such a so-called "catastrophic bifurcation" or "discontinuous transition to extinction" is a prerequisite for evolutionary suicide. A simple geometric explanation of this necessary condition is given in Box 11.5.

This result allows us to distinguish strictly between mere evolutionary deterioration and actual evolutionary suicide:

- Evolutionary deterioration implies that evolution by natural selection gradually drives a population to lower and lower densities until it is eventually removed by demographic or environmental stochasticity. The first example above, by Matsuda and Abrams (1994a), is of this kind.

- By contrast, evolutionary suicide implies that evolution by natural selection drives a population toward a catastrophic bifurcation through which its density abruptly decreases to zero. Notice that it is the evolutionary time scale on which this extinction is abrupt; on the ecological time scale, of course, a decrease in 
Box 11.5 Transcritical bifurcations exclude evolutionary suicide

Wherever a population goes through a continuous transition to extinction it cannot undergo evolutionary suicide. For simplicity, we show this for cases in which the population's density $N$ and its adaptive trait $x$ are both one-dimensional. The generic continuous transition to extinction is then the so-called transcritical bifurcation, in which a positive equilibrium and the extinction equilibrium exchange their stability at a critical trait value $x_{c}$.

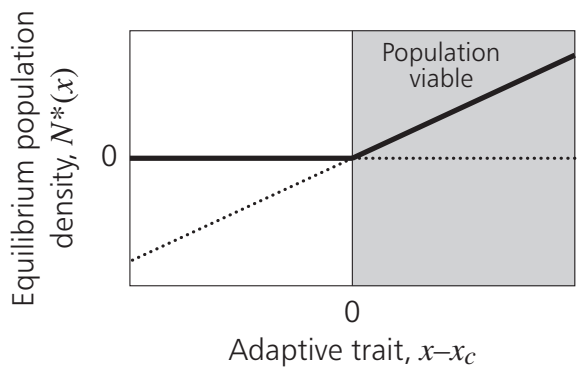

In the vicinity of the critical trait value $x_{c}$, population dynamics that exhibit a transcritical bifurcation can always be written as $\frac{d}{d t} N=r\left[\left(x-x_{c}\right)-N / K\right] N$, where $K>0$ scales $N$ and $r>0$ scales $\frac{d}{d t} N$ (up to re-orientation of the direction of $x$; Guckenheimer and Holmes 1997, p. 145). With the per capita growth rate of a mutant trait value $x^{\prime}$ in an environment with population density $N$ thus being given by $r\left[\left(x^{\prime}-x_{c}\right)-N / K\right]$, and with the equilibrium population density of a resident population with trait value $x=x_{c}$ vanishing $(N=0)$, we obtain the invasion fitness $f\left(x^{\prime}, x_{c}\right)=r\left(x^{\prime}-x_{c}\right)$ for the rare mutant that competes with the critical resident. In addition, the consistency condition $f(x, x)=0$ for ecological equilibrium has to be fulfilled for all $x$ (see Box 11.2). If we now make the generic assumption that $f\left(x^{\prime}, x\right)$ has a leading linear order around $x^{\prime}=x_{c}$ and $x=x_{c}$, that is, $f\left(x^{\prime}, x\right)=$ $c^{\prime} x^{\prime}+c x$, we can determine the coefficients $c^{\prime}$ and $c$ from the two constraints (1) $f\left(x^{\prime}, x_{c}\right)=r\left(x^{\prime}-x_{c}\right)$ for all $x^{\prime}$ and (2) $f(x, x)=0$ for all $x$, which yield $f\left(x^{\prime}, x\right)=$ $r\left(x^{\prime}-x\right)$. For the selection gradient (Box 11.3) we thus obtain $g(x)=r$, which is always positive. This means that adaptive evolution takes $x$ away from $x_{c}$ by making it larger, and thus increases the equilibrium population size from $N^{*}\left(x_{c}\right)=0$ to $N^{*}(x)=\left(x-x_{c}\right) K$. Therefore, adaptive evolution in this system cannot cause evolutionary suicide by driving $x$ toward the critical trait value $x_{c}$.

The above reasoning can be collapsed to a glance at an illustration of the local geometry, as sketched below.

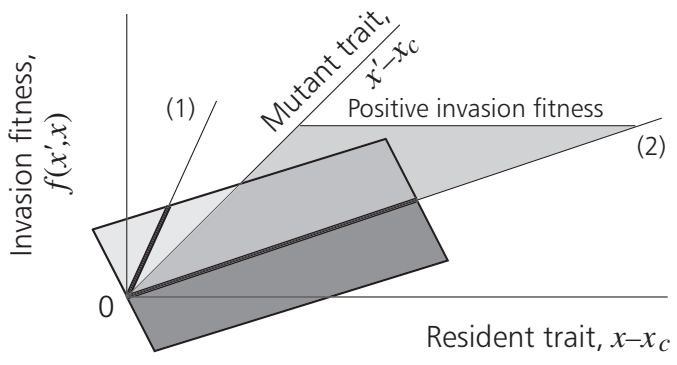

Since the plane that represents the invasion fitness $f\left(x^{\prime}, x\right)$ is constrained to pass through the two straight lines that represent constraints (1) and (2), the region $x^{\prime}>x$ has a positive invasion fitness. Thus evolution increases $x$, moving it away from $x_{c}$. 
population density always takes a while to result in extinction. The third example above, by Gyllenberg et al. (2002), is of this kind [as is the second example, by Dercole et al. (2002), although it involves a catastrophic bifurcation that does not lead to immediate extinction].

The important role played by demographic and environmental stochasticity in evolutionary deterioration means that such processes may also be referred to loosely as stochastic evolutionary suicide. The same applies to an evolutionary collapse that exposes a population to a high risk of accidental extinction. Another form of stochastic evolutionary suicide, driven by mutational stochasticity rather than by demographic or environmental stochasticity, and not discussed here, can occur in higher-dimensional trait spaces (Parvinen and Dieckmann, in press).

\section{Further examples of evolutionary suicide}

Another example of evolutionary suicide is driven by the evolution of dispersal rates (Gyllenberg et al. 2002). The ecological model of structured metapopulations that underlies this example was introduced by Gyllenberg and Metz (2001) and Metz and Gyllenberg (2001). It considers a large number of identical patches of habitable environment. Each patch can support a local population. Patches are connected by dispersal, and individuals leave their patch at a rate that can evolve through mutation and selection. Dispersal risk is defined as the probability that a dispersing individual will not survive until it settles down again in a patch. Local populations may go extinct as a result of catastrophes. At least two scenarios, which involve two different kinds of Allee effects, can then cause evolutionary suicide in this model. First, evolutionary suicide occurs when small local populations have a negative intrinsic growth rate and thus can only persist through immigration from other patches: when a high dispersal risk then selects for a low dispersal rate, adaptation drives the metapopulation to extinction. Second, evolutionary suicide can also occur when the rate at which local populations are wiped out by catastrophes increases as the population size decreases: again, a high dispersal risk makes dispersal unattractive for individuals, even though the population as a whole depends on this dispersal. This selection pressure results in an abrupt extinction of the metapopulation when the dispersal rates falls below a critical level. A more detailed discussion of this family of models is provided in Chapter 14.

For adaptive evolution that involves kin selection, Le Galliard et al. (2003) observed evolutionary suicide caused by the adaptive dynamics of altruism. In this model, three selective forces act on an adaptive trait that measures the level of altruistic investment:

1. Direct, physiological cost of investing more in the altruistic behavior;

2. Indirect benefit of locally interacting with more altruistic individuals; and

3. Indirect cost of locally saturating the habitat.

Since locally interacting individuals often share a common ancestry, the second selection pressure involves kin selection. The third selection pressure turns out to be negligible because demographic stochasticity and individual mobility tend to 
reduce local crowding. Mobility has a cost, however, and the population cannot sustain itself at a high mobility without a substantial degree of altruism between individuals. The combination of high mobility and a high ambient level of altruism creates the ideal conditions for "cheaters" to invade - phenotypes that invest slightly less in altruism and yet reap the full benefits provided by the resident, more altruistic individuals. This causes the population to evolve toward a state in which the population's level of altruism is no longer sufficient to ensure its persistence, resulting in evolutionary suicide.

Evolutionary suicide can also be expected when adaptive evolution involves sexual selection (Kirkpatrick 1996). Mating preferences can establish a trait even if it has negative side effects on an individual's survival. A gene for a preferred trait that is expressed in both sexes will spread if its fitness gain through male mating success more than offsets its survival cost evaluated over males and females (Kirkpatrick 1982). Thus, adaptive evolution can establish traits that have negative effects on female reproductive success, and hence on the population's reproductive output. We expect, and often see, the evolution of modifiers suppressing the expression of those genes in females that give a fitness advantage only to males, even though sexual selection can cause the evolution of traits that decrease population viability. This feature of sexual selection had already been realized by Darwin (and presumably was one of the reasons why he attributed so much emphasis to the distinction between natural and sexual selection).

A recent study by Ernande et al. (2002) shows that selection-induced extinction can, in principle, also happen in the context of exploited living resources, where these are modeled realistically. The model considers a physiologically structured population in which individuals continually age and grow in body size. On reaching a size threshold, they turn from larvae dispersed only passively into juveniles able actively to select their local environment. These local environments differ in the growth and mortality rates they induce. When the growth trajectories of individuals reach the maturation reaction norm, represented as a function that describes maturation size as dependent on maturation age, juveniles turn into adults and start to reproduce at a rate that increases with their body size. In this model the shape of the maturation reaction norm is the evolving trait. Ernande et al. (2002) show that when the evolving population is exposed to a harvesting regime that extracts biomass at a constant rate, the maturation reaction norm evolves so as to allow individuals to mature at younger ages and smaller sizes. At a certain point, this adaptation may cause the entire population to become extinct - a phenomenon of evolutionary suicide that is especially worrisome in the context of commercially exploited fish stocks.

\section{Evolutionary suicide in sexual populations}

A factor that could prevent evolutionary suicide in sexual populations is the premature depletion of additive genetic variance (Matsuda and Abrams 1994a, 1994b). If the additive genetic variance approaches zero as the trait value approaches the suicidal threshold, the evolutionary process will be much decelerated. However, 
unless mutations cease to induce genetic variance, eventual evolutionary suicide remains inevitable.

At the opposite end of the spectrum, a surplus of phenotypic variance may prevent evolutionary suicide. This can be understood as follows. When a broad phenotypic distribution approaches a suicidal threshold, it extends its head tail beyond the population's viability domain. The loss of individuals in this tail then affects the selective pressures that act on the rest of the population. In particular, the release of density regulation through very low reproductive success in the head tail may boost the reproductive success of individuals in the rear tail. It turns out that this source-sink dynamics across a population's viability boundary can allow the population to hover temporarily at the brink of extinction. The smaller the population's phenotypic variance, the closer it approaches extinction. This places an extra premium on maintaining the genetic variance of populations threatened by evolutionary suicide: once their variance is sufficiently depleted, their extinction is imminent.

Box 11.6 shows how evolutionary suicide is expected to occur in sexual populations and, in particular, how the underlying genetics could interfere with the ecology of evolutionary suicide as outlined above.

\section{Extinction driven by coevolutionary dynamics}

Also, coevolutionary dynamics can cause extinctions. Some early treatment, which still excludes the effects of intraspecific frequency-dependent selection, is given in Roughgarden (1979).

Dieckmann et al. (1995) considered an example of predator-prey coevolution in which the predator's extinction is caused by the prey's adaptation. In this model, the phenotype of a predator has to remain sufficiently close to that of its prey for the predator's harvesting efficiency to remain high enough to ensure predator survival. This may reflect the need for a match between, for example, prey size and the dimensions of the predator's feeding apparatus. Thus, whenever evolution in the prey takes its phenotype too far away from the predator's matching phenotype, harvesting efficiency drops below a critical level, and so causes the predator to become extinct.

Notice that in all cases in which such a transition to extinction is gradual (rather than discontinuous), evolutionary suicide cannot contribute to the extinction (Box 11.5). In addition, gradual extinction causes mutation-limited phenotypic evolution in the dwindling species to grind to a halt, since fewer and fewer individuals are around to give birth to the mutant phenotypes that fuel the adaptive process (Box 11.3). This stagnation renders the threatened species increasingly defenseless by depriving it of the ability to counteract the injurious evolution of its partner by a suitable adaptation of its own. For these two reasons, gradual coevolutionary extinction is driven solely by adaptation in the coevolving partner(s). The situation is different, of course, when a transition to extinction is discontinuous: in such cases, processes of evolutionary suicide and "coevolutionary murder" may conspire to oust a species from the coevolving community. 
Box 11.6 Evolutionary suicide in sexual populations

The dynamics of sexual populations differ in several respects from those considered in asexual models of evolutionary suicide. In particular, sexual populations are typically polymorphic, which has two important implications:

- First, compared with a monomorphic asexual population that features the same mean value of the adaptive trait, variance corrections to the model's invasion fitness are bound to arise. These corrections can affect the population size predicted for a given trait value, the critical trait values at which evolutionary suicide is expected, and the selection gradient. Occasionally, a changed selection gradient may even enable evolution away from the extinction boundary.

- Second, a polymorphic population may hover at the brink of extinction, because the death of individuals in the population's tail that extends beyond the extinction boundary may enhance reproductive success in the remaining population.

The two illustrations below show results for a sexual model of evolutionary suicide; to our knowledge this is the first time such an analysis has been carried out. Based on an adaptive trait $x$, per capita birth rates are given by $b(x)=b_{0} /\left(1+K_{1 / 2} / N\right)$, and per capita death rates by $d(x)=d_{0}+N_{\text {eff }}(x) / K(x)$. Here, $b_{0}$ and $d_{0}$, respectively, denote the intrinsic birth and death rates, $N$ the population's total size, and $K_{1 / 2}$ the population size at which $b$ drops to $\frac{1}{2} b_{0}$ through an Allee effect. The death rate is increased by asymmetric competition, with the sum in $N_{\text {eff }}(x)=\sum_{i} \alpha\left(x_{i}-x\right)$ extending over all individuals, and the competitive effect of an individual with trait value $x_{i}$ on an individual with trait value $x$ given by $\alpha\left(x_{i}-x\right)=2 /\left[1+e^{-\left(x_{i}-x\right) / w}\right]$, where $w$ determines the degree of competitive asymmetry. Asymmetric competition thus favors individuals with larger values of the adaptive trait $x$. The population's carrying capacity is trait dependent and given by a normal function, $K(x)=K_{0} \exp \left(-\frac{1}{2} x^{2} / \sigma_{K}^{2}\right)$, which thus favors individuals with intermediate values of the adaptive trait $x$. For the illustrations below, parameters are set to $b_{0}=1, d_{0}=0.2, K_{0}=2000, K_{1 / 2}=200, \sigma_{K}=1$, and $w=0.2$. The adaptive trait $x$ is polygenic, determined by $n=10$ equivalent diploid loci with additive effects and free recombination. Loci can either be diallelic, with allelic values +1 and -1 , or they can feature a continuum of alleles. The set of trait values in the diallelic model is scaled to $-2<x<+2$, with an analogous scaling applied to the infinite-allele model. Mutations occur at a probability of $u=10^{-3}$ per locus and, in the case of continuous allelic values, are distributed normally with standard deviation $\sigma=0.2$.
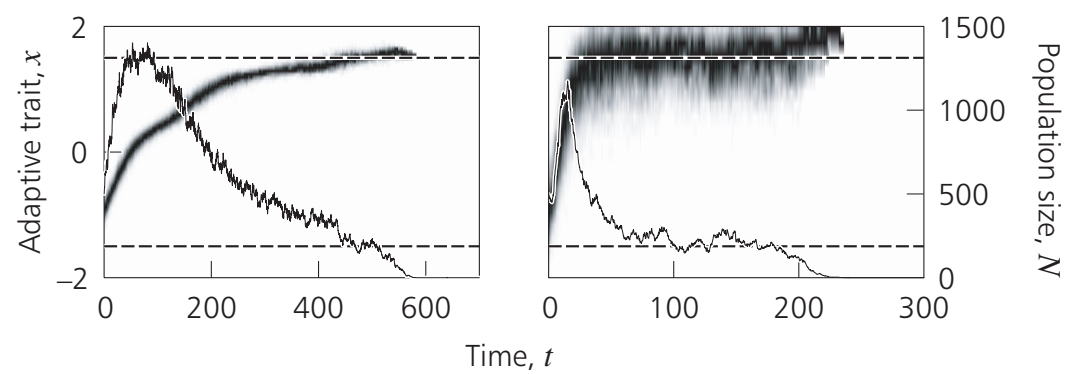

continued 


\section{Box 11.6 continued}

The left panel on the preceding page is based on the infinite-allele model and shows how the polymorphic distribution of adaptive trait values, depicted by gray scales, starts out on one side of the carrying capacity's peak at $x=0$ and then, driven by asymmetric competition, evolves toward and beyond this peak, until it reaches the extinction boundary at about $x=1.5$. The model's two extinction boundaries are depicted by dashed lines (notice that, since selection drives the population away from the lower boundary, no evolutionary suicide can occur there). The black continuous curve shows the changes in actual population size that result from the trait's evolution. The dynamics of evolutionary suicide in this model is thus very similar to that predicted by the corresponding asexual model. The right panel shows exactly the same situation, except that the diallelic model is now being considered. The different genetic architecture that underlies the adaptive trait $x$ imposes a much larger phenotypic variance on the population throughout all phases of its evolution. With just a few diallelic loci, this phenotypic variance is now so large that the population lingers for a while at the brink of extinction, before perishing eventually.

We can thus conclude that - except for some quantitative corrections and for the extra potential of populations to hover temporarily at the brink of extinction - the phenomenon of selection-driven extinction appears to apply just as well to sexual populations as it does to the asexual populations investigated in earlier studies.

Further examples of coevolution-driven extinction are provided in Chapter 16 for coevolving communities that exhibit both mutualistic and competitive interactions.

\section{Summary}

Evolutionary suicide occurs for a rich variety of ecological settings and appears to be robust to variations in the underlying system of inheritance. Even if evolutionary suicide does not occur, the related phenomena of persistent evolutionary deterioration or of an abrupt collapse toward perilously low densities are possible. Also, coevolution can bring about a species' demise. Thus, phenomena in which the adaptive process itself harms an evolving species or community are by no means peculiar outcomes of particularly rigged ecological models.

A question of acute interest in the context of population management is to identify the circumstances through which environmental change can expose a population to the threat of evolutionary deterioration, collapse, or suicide. We address this issue in the following section.

\subsection{Adaptive Responses to Environmental Change}

Populations exposed to environmental change usually experience altered selection pressures acting on their traits. If the population had enough time to adapt to the prevailing environmental conditions before the evolutionary change, with the result that selection had become stabilizing, it typically experiences a qualitative 
change to directional selection during and after the environmental change. Classic models of such situations are based on the notion of a fitness maximum that gradually shifts its position in trait space. The primary question is then whether or not the evolving population can respond quickly enough to the new directional selection pressures for it to track the shifting maximum and thus to persist despite the threatening change in its environment. Questions of this kind are best analyzed using techniques of quantitative genetics, and are discussed in detail in Chapter 10. Here we take a broader perspective and consider more general (and intricate) patterns of interplay between environmental change, adaptive evolution, and ecological viability.

\section{Ecology-evolution-environment diagrams}

A geometric approach to the interplay of ecology, evolution, and environment is facilitated by focusing attention on conditions that imply population extinction. For this purpose we consider those phenotypic values $x$ that allow a population to be viable under environmental conditions $e$. Combinations $(x, e)$ that do not allow for this imply population extinction. Such a focus on extinction conditions conveniently removes the population size $N$ from the graphic considerations below, which renders the resultant diagrams much easier to read.

To describe the evolutionary dynamics for viable combinations $(x, e)$ of phenotypes and environmental conditions, we can utilize the pairwise invasibility plots introduced in Box 11.2, which allow us to consider all kinds of density- and frequency-dependent selection. Figure 11.3a shows a sample sequence of pairwise invasibility plots that illustrates how they may change when environmental conditions are altered:

Initially, an evolutionary attractor (technically speaking, a convergence-stable evolutionary singularity) coexists with an evolutionary repellor, both situated away from an extinction region of trait values that render the population unviable.

- As environmental conditions change, the two evolutionary singularities approach each other.

- Eventually, they collide.

- Directional selection then drives the evolving phenotype into the extinction region, causing evolutionary suicide.

Figure $11.3 \mathrm{~b}$ shows how this very same sequence of events can be depicted in a single diagram, employing three characteristic features:

- Arrows show the direction of selection;

- Line styles indicate the different types of evolutionary singularity; and

- Shading shows the extinction regions.

We refer to such plots as ecology-evolution-environment diagrams, or $\mathrm{E}^{3}$ diagrams for short: the environmental and evolutionary components of change 


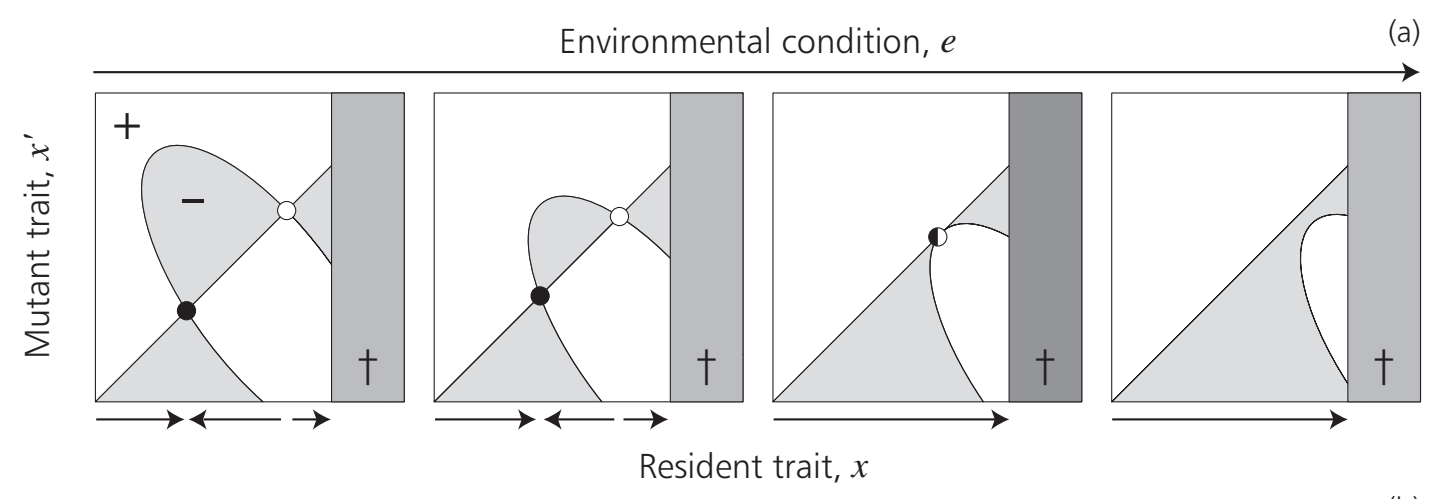

(b)

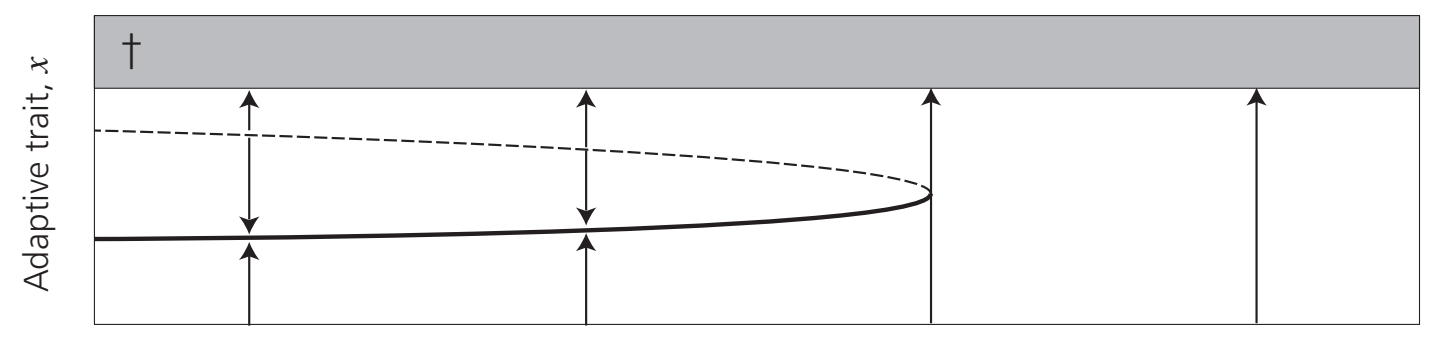

Environmental condition, $e$

Figure 11.3 Interplay between ecological, evolutionary, and environmental change. (a) Pairwise invasibility plots that show the collision and resultant disappearance of an evolutionary attractor and repellor, which lead to induced evolutionary suicide as environmental conditions are varied toward the right. Light gray regions correspond to negative invasion fitness; the extinction region is shown in dark gray. Evolutionary attractors and repellors are depicted by filled circles and open circles, respectively. Short arrows indicate the direction of selection. (b) Ecology-evolution-environment diagram ( $\mathrm{E}^{3}$-diagram) that depicts the same situation as in (a). Arrows show the direction of selection, line styles indicate the type of evolutionary singularity, and shading shows the extinction region. Continuous (dashed) curves indicate evolutionary attractors (repellors), while thick (thin) curves indicate evolutionarily stable (unstable) singularities.

are represented along the horizontal and vertical axes, respectively, while the ecology furnishes the shown selection pressures and determines the extinction regions throughout which the population is not viable. Comparing Figures 11.3a and 11.3b suggests that a single $\mathrm{E}^{3}$-diagram is more immediately comprehensible than sequences of pairwise invasibility plots, while, as long as we are content to consider gradual evolution, they contain the same salient information.

$\mathrm{E}^{3}$-diagrams have much in common with those regularly used in the classic bifurcation theory of dynamic systems (e.g., Kuznetsov 1995; Guckenheimer and Holmes 1997) - yet they acquire essential extra complexity because of two additional features: first, the incorporation of the extinction region, and second, the distinction between evolutionarily stable and unstable singularities. In the classic theory, only convergence stability would be considered, and consequently only evolutionary attractors and repellors would be discriminated.

We notice in passing that, if, for a particular study, a need were to arise to retain more ecological information in $\mathrm{E}^{3}$-diagrams, then contour lines of, for example, 
the equilibrium (or, alternatively, the time-averaged) population size $N$ that results for particular combinations of phenotypes $x$ and environmental conditions $e$ could be added to the diagrams readily. Likewise, if the resident population dynamics can undergo bifurcations, it would be instructive to add the corresponding bifurcation boundaries to the $\mathrm{E}^{3}$-diagram.

We now utilize $\mathrm{E}^{3}$-diagrams to highlight a number of phenomena that are of general relevance to evolutionary conservation biology.

\section{Ecological and evolutionary penalties of environmental change}

$\mathrm{E}^{3}$-diagrams can be used to elucidate different conservation perils associated with fast or large environmental change. Even for the simplest case - in which the dynamics of population size, phenotype, and environment can all be described as one-dimensional - at least three time scales are involved in a population's exposure to environmental change; these characterize the rates of change in population size, phenotype, and environmental condition. For most organisms we can safely assume that population dynamics occur faster than evolutionary dynamics. Relative to these two time scales, environmental change can then be faster, intermediate, or slower.

Figures 11.4a to $11.4 \mathrm{c}$ illustrate three different ways in which fast environmental change can cause population extinction:

- In Figure 11.4a, environmental change occurs so rapidly that it outpaces both the population dynamics and the evolutionary dynamics of the affected population. The figure shows how environmental change takes the population right into an extinction region (Point A), where the population gradually diminishes in size and eventually perishes.

- Figure 11.4a also shows what happens when environmental change occurs at an intermediate time scale, rendering it slower than the population dynamics, but faster than the evolutionary dynamics. Under these circumstances the population becomes extinct as soon as environmental change forces it to trespass into the extinction region: population extinction thus occurs right at the region's boundary (Point B).

- In Figure 11.4b, environmental change occurs at a time scale commensurable with that of evolutionary dynamics. The figure shows that even such a situation can still lead to population extinction: as soon as the environmental change forces the population across the separatrix curve that corresponds to the evolutionary repellor, the course of directional evolution is reversed and the population steers toward evolutionary suicide.

- Finally, in Figure 11.4c environmental change is sufficiently slow for evolutionary rescue (Gomulkiewicz and Holt 1995) to become feasible. Such a situation allows the population to track its evolutionary attractor, which in the illustrated case saves the population from extinction.

When environmental change is abrupt, the amount of change becomes key to predicting the fate of the exposed population. Such situations can also be analyzed 
(a)

(b)

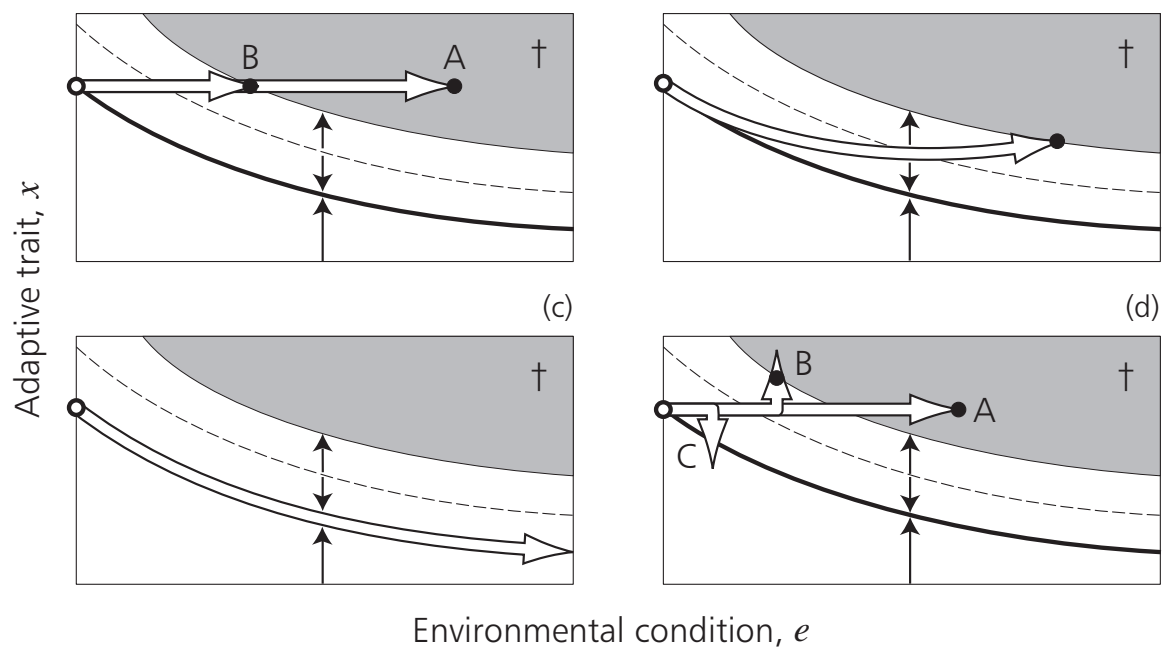

Figure 11.4 Ecological and evolutionary penalties of fast or large environmental change. Elements of the $\mathrm{E}^{3}$-diagrams are as in Figure 11.3b. Open circles show the population's state before environmental change occurs, white arrows depict the resultant trajectories, and filled circles indicate where the population becomes extinct. (a) If environmental change is faster than the population dynamics, the population perishes in the interior of the extinction region (Point A). If environmental change is slower than the population dynamics, but faster than the evolutionary dynamics, the population vanishes at the border of the extinction region (Point B). (b) If environmental change occurs at the same time scale as that of the evolutionary dynamics, the population may still undergo induced evolutionary suicide once it is trapped beyond an evolutionary repellor (dashed curve). (c) If environmental change is sufficiently slow, evolutionary rescue may occur. (d) Consequences of abrupt environmental changes of different magnitudes. An ecological penalty occurs if the environmental change takes the population into the extinction region (Trajectory A), whereas an evolutionary penalty occurs if the environmental change takes it beyond the evolutionary repellor (Trajectory B). If the environmental change is small enough, the population may be rescued by adaptation (Trajectory $\mathrm{C}$ ).

conveniently using $\mathrm{E}^{3}$-diagrams. Retaining the same setting as for Figures $11.4 \mathrm{a}$ to $11.4 \mathrm{c}$, Figure $11.4 \mathrm{~d}$ illustrates two fundamentally different ways in which large and abrupt environmental changes can cause population extinction:

- A large environmental change settles the population right in the extinction region, which implies its demise through the ensuing population dynamics (Trajectory A).

- An intermediate environmental change moves the population beyond the separatrix given by the position of the evolutionary repellor, which causes its extinction through evolutionary suicide (Trajectory B).

- By contrast, a small environmental change allows the population to stay on the safe side of the evolutionary separatrix, and thus enables it to undergo evolutionary rescue (Trajectory $\mathrm{C}$ ). 
These simple examples highlight conceptually distinct penalties associated with environmental change: an ecological penalty occurs when a population's viability is forfeited as a direct consequence of environmental change (Points A and B in Figure 11.4a; Trajectory A in Figure 11.4d), whereas an - often less obvious - evolutionary penalty is incurred when environmental change compromises a population's ability to evolve out of harm's way (Figure 11.4b; Trajectory B in Figure 11.4d).

\section{Evolutionary rescue, trapping, and induced suicide}

$\mathrm{E}^{3}$-diagrams are also useful to depict the phenomena of evolutionary rescue and trapping introduced in Box 1.4. In fact, the left and middle plots in Box 1.4 can be interpreted as $\mathrm{E}^{3}$-diagrams if we take their horizontal axis to measure environmental condition, rather than time. Evolutionary rescue can occur when an evolutionary attractor escapes an encroaching extinction region as the environmental conditions are changed. Similarly, evolutionary trapping - in its simplest form (see below) - requires that an evolutionary attractor collide with an extinction region as environmental conditions are changed.

In contrast to evolutionary rescue and trapping, evolutionary suicide can occur in the absence of any extrinsic environmental change, as it is intrinsically driven by the feedback between an evolving population and its environment. The fingerprint of evolutionary suicide in $\mathrm{E}^{3}$-diagrams is directional selection pointing toward an extinction region as, for example, in the right part of Figure 11.3b.

Evolutionary suicide, however, is involved critically in another phenomenon we need to understand to assess a population's response to environmental change. Figure 11.3b illustrates this scenario, which we call induced evolutionary suicide: an evolutionary attractor collides with an evolutionary repellor, such that a population that is tracking the attractor as environmental conditions are changing suddenly becomes exposed to directional selection toward the extinction region, and hence undergoes evolutionary suicide. Here it is the environmental change that abruptly creates the conditions that lead to evolutionary suicide.

\section{More complex forms of evolutionary trapping}

Figure 11.5a illustrates how induced evolutionary suicide can result in a more complex form of evolutionary trapping. Here an evolutionary attractor again vanishes in collision with a repellor. Now, however, there is a range of environmental conditions in which two attractor-repellor pairs coexist. This means that, if a large jump occurs in its phenotype, the population could survive environmental conditions that change toward the right by shifting to the lower attractor. Gradual phenotypic change, however, keeps the population trapped at the upper attractor, and results in its inevitable demise.

A much more benign (and simple) situation is depicted in Figure 11.5b. Here small environmental change results in a large shift of an evolutionary attractor, which obviously makes it difficult for gradual evolution to catch up with the required pace of phenotypic change. Such a situation could thus be described as an 
(a)

(b)

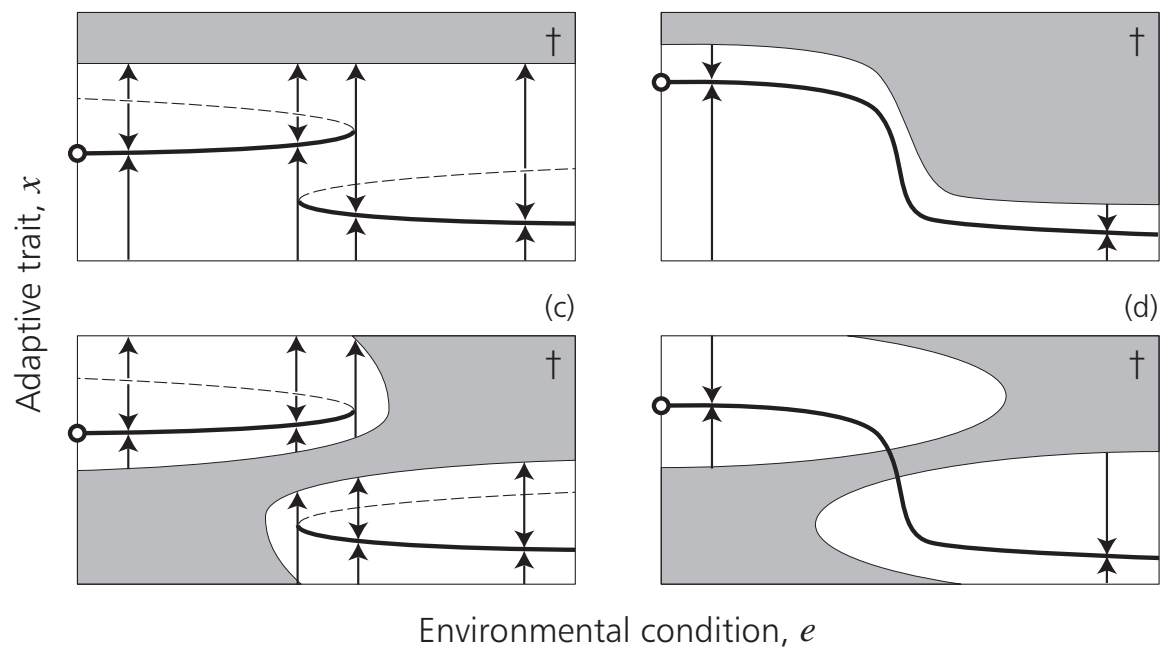

Figure 11.5 Examples of more complex forms of evolutionary trapping. Elements of the $\mathrm{E}^{3}$-diagrams are as in Figure 11.3b. Open circles show the population's state before environmental change shifts conditions to the right. (a) When one evolutionary attractor vanishes in a collision with a repellor, the population can only survive by a large phenotypic jump across a fitness valley to the alternative evolutionary attractor. (b) When the positions of the evolutionary attractor and the extinction region undergo substantial changes in response to a small environmental change, the population can survive only through a particularly swift evolution. In such situations, the population easily becomes trapped by limitations on its pace of adaptation. The settings in (c) and (d) are the same as those in (a) and (b), respectively, but with the two evolutionary attractors now separated by the extinction region.

evolutionary bottleneck, where, at some stage, only swift adaptation could rescue the threatened population.

The scenarios in Figures $11.5 \mathrm{a}$ and $11.5 \mathrm{~b}$ can be exacerbated considerably if the extinction region takes a more complex and expansive shape. Such cases are illustrated in Figures $11.5 \mathrm{c}$ and 11.5d. In both cases, gradual evolution cannot rescue the population. The shift of the population's phenotype to the safe evolutionary attractor now not only requires it to trespass through a fitness valley (as in Figure 11.5a) or particularly rapid evolution (as in Figure 11.5b), but also gradual evolutionary change toward the safe attractor takes the population into the extinction region, and thus completely forestalls gradual evolution as a sufficient evolutionary response to the imposed environmental change.

The latter two scenarios may look complex, but are not as improbable as one perhaps would wish to think: Section 14.4 describes dispersal evolution in response to landscapes changes and presents, in Figure 14.10b, a result of the type depicted in Figures $11.5 \mathrm{c}$ and $11.5 \mathrm{~d}$. In Chapter 14, also the results shown in Figures $14.4,14.11,14.12$, and 14.13 showcase the use of $\mathrm{E}^{3}$-diagrams in understanding the conservation implications of dispersal evolution. A particularly intriguing finding in this context is that induced evolutionary suicide can result from environmental conditions that become less severe, as illustrated in Figure 14.13b. 


\subsection{Concluding Comments}

For a long time, the common wisdom in evolutionary ecology was that adaptive evolution by natural selection should maximize some measure of fitness, and hence population viability. In this chapter we discuss several fundamental shortcomings that restrict the scope of this earlier approach (Section 11.2), and investigate the consequences of a more realistic understanding of adaptive evolution (Section 11.3) for the origin and loss of biodiversity (Sections 11.4 and 11.5), as well as for the response of threatened populations to environmental change (Section 11.6).

We show that it is only under very special circumstances that adaptive evolution follows an optimization principle, maximizing some measure of fitness. This special case occurs when the environmental feedback loop is one-dimensional and monotonic - and even then population viability cannot always be expected to be maximized: as described in Section 11.2, adaptive evolution that follows an optimization principle can drive a population to extinction. Such evolutionary suicide turns out to be a common phenomenon in more realistic models that incorporate frequency- and density-dependent selection, and must therefore be expected to play a major role in the loss of biodiversity. The other facet of non-optimizing adaptive evolution is its role in promoting biodiversity by means of evolutionary rescue and evolutionary branching, which result in the maintenance or even enhancement of biodiversity. A suite of new theoretical tools is thus now in place to translate ecological knowledge of the interaction of populations with their environment into quantitative predictions about the evolving diversity of ecological communities.

The analysis of adaptive responses to environmental change raises new challenges for conservation biology and evolutionary theory. We have introduced $\mathrm{E}^{3}$ analysis as a tool to investigate and predict the conservation perils associated with environmental changes that unfold on different time scales. $E^{3}$-diagrams summarize the salient features of series of pairwise invasibility plots obtained for gradually changing environmental conditions and enable easy graphic interpretation. The use of such $\mathrm{E}^{3}$-diagrams and, more generally, of adaptive dynamics models in changing environments provide a synthetic approach to the dramatic consequences of adaptive evolution on biodiversity in a changing world. Indeed, it would seem advisable to extend medium-term conservation efforts based on traditional models of population extinction by taking advantage of the now-available new tools to link ecological and evolutionary insights.

Acknowledgments We are grateful to Fabio Dercole, Jean-François Le Galliard, Hans Metz, Kalle Parvinen, and Sergio Rinaldi for interesting discussions. Financial support for this study was received from the Austrian Federal Ministry of Education, Science, and Cultural Affairs, and the Austrian Science Fund to Ulf Dieckmann; from the French Ministère de l'Education Nationale de la Recherche et des Technologies ("ACI Jeunes Chercheurs 2000" and "ACI Bioinformatique 2001"), and from the French Ministère de l'Aménagement du Territoire et de l'Environnement (Programme "Invasions Biologiques") to Régis Ferrière; and from the European Research Training Network ModLife (Modern Life-History Theory and its Application to the Management of Natural Resources), funded through the Human Potential Programme of the European Commission, to Ulf Dieckmann and Régis Ferrière. 


\section{References}

References in the book in which this chapter is published are integrated in a single list, which appears on pp. 365-410. For the purpose of this reprint, references cited in the chapter have been assembled below.

Abrams PA, Matsuda H \& Harada Y (1993). Evolutionarily unstable fitness maxima and stable fitness minima of continuous traits. Evolutionary Ecology 7:465-487

Allen JC, Schaffer WM \& Rosko D (1993). Chaos reduces species extinction by amplifying local-population noise. Nature 364:229-232

Bürger R (1998). Mathematical properties of mutation-selection models. Genetica 103:279-298

Bürger R \& Bomze IM (1996). Stationary distributions under mutation-selection balance: Structure and properties. Advances in Applied Probability 28:227-251

Bush GL (1969). Sympatric host race formation and speciation in frugivorous flies of the genus Rhagoletis (Diptera: Tephritidae). Evolution 23:237-251

Calow P (1999). Encyclopedia of Ecology and Environmental Management. Oxford UK: Blackwell Publishing

Case TJ (1999). An Illustrated Guide to Theoretical Ecology. Oxford, UK: Oxford University Press

Charnov E (1993). Life History Invariants: Some Explorations of Symmetry in Evolutionary Ecology. Oxford, UK: Oxford University Press

Darwin C (1859). On the Origin of Species. London, UK: John Murray

Dennis B (1989). Allee effects: Population growth, critical density, and the chance of extinction. Natural Resource Modeling 3:481-538

Dercole F, Ferrière R \& Rinaldi S (2002). Ecological bistability and evolutionary reversals under asymmetrical competition. Evolution 56:1081-1090

Dieckmann U (1994). Coevolutionary Dynamics of Stochastic Replicator Systems. Jülich, Germany: Central Library of the Research Center Jülich

Dieckmann U (2002). Adaptive dynamics of pathogen-host interactions. In Adaptive Dynamics of Infectious Diseases: In Pursuit of Virulence Management, eds. Dieckmann U, Metz JAJ, Sabelis MW \& Sigmund K, pp. 39-59. Cambridge, UK: Cambridge University Press

Dieckmann U \& Doebeli M (1999). On the origin of species by sympatric speciation. Nature 400:354-357

Dieckmann U \& Doebeli M (2004). Adaptive dynamics of speciation: Sexual populations. In Adaptive Speciation, eds. Dieckmann U, Doebeli M, Metz JAJ \& Tautz D, pp. 76-111. Cambridge, UK: Cambridge University Press

Dieckmann U \& Heino M. The adaptive dynamics of function-valued traits. Unpublished

Dieckmann U \& Law R (1996). The dynamical theory of coevolution: A derivation from stochastic ecological processes. Journal of Mathematical Biology 34:579-612

Dieckmann U, Marrow P \& Law R (1995). Evolutionary cycling of predator-prey interactions: Population dynamics and the Red Queen. Journal of Theoretical Biology 176:91102

Dieckmann U, Doebeli M, Metz JAJ \& Tautz D, eds. (2004). Adaptive Speciation. Cambridge, UK: Cambridge University Press

Doebeli M \& Dieckmann U (2000). Evolutionary branching and sympatric speciation caused by different types of ecological interactions. The American Naturalist 156:S77S101 
Doebeli M \& Dieckmann U (2003). Speciation along environmental gradients. Nature 421:259-264

Doebeli M \& Dieckmann U (2004). Adaptive dynamics of speciation: Spatial structure. In Adaptive Speciation, eds. Dieckmann U, Doebeli M, Metz JAJ \& Tautz D, pp. 140-167. Cambridge, UK: Cambridge University Press

Drake JA, Mooney HA, di Castri F, Groves RH, Kruger FJ, Rejmanek M \& Williamson M, eds. (1989). Biological Invasions: A Global Perspective. Brisbane, Australia: John Wiley \& Sons

Emlen JM (1987). Evolutionary ecology and the optimality assumption. In The Latest on the Best, ed. Dupre J, pp. 163-177. Cambridge, MA, USA: MIT Press

Ernande B, Dieckmann U \& Heino M (2002). Fisheries-induced changes in age and size at maturation and understanding the potential for selection-induced stock collapse. In The Effects of Fishing on the Genetic Composition of Living Marine Resources, ICES CM 2002/Y:06. Copenhagen, Denmark: International Council for the Exploration of the Seas

Eshel I (1983). Evolutionary and continuous stability. Journal of Theoretical Biology 103:99-111

Eshel I \& Motro U (1981). Kin selection and strong evolutionary stability of mutual help. Journal of Theoretical Population Biology 19:420-433

Felsenstein J (1981). Skepticism towards Santa Rosalia, or why are there so few kinds of animals? Evolution 35:124-238

Ferrière R (2000). Adaptive responses to environmental threats: Evolutionary suicide, insurance, and rescue. Options Spring, pp. 12-16. Laxenburg, Austria: International Institute for Applied Systems Analysis

Ferrière R \& Gatto M (1995). Lyapunov exponents and the mathematics of invasion in oscillatory or chaotic populations. Theoretical Population Biology 48:126-171

Fisher RA (1930). The Genetical Theory of Natural Selection. Oxford, UK: Clarendon Press

Frank SA \& Slatkin M (1992). Fisher's fundamental theorem of natural selection. Trends in Ecology and Evolution 7:92-95

Gatto M (1993). The evolutionary optimality of oscillatory and chaotic dynamics in simple population-models. Theoretical Population Biology 43:310-336

Geritz SAH \& Kisdi É (2000). Adaptive dynamics in diploid sexual populations and the evolution of reproductive isolation. Proceedings of the Royal Society of London B 267:1671-1678

Geritz SAH, Metz JAJ, Kisdi É \& Meszéna G (1997). Dynamics of adaptation and evolutionary branching. Physical Review Letters 78:2024-2027

Geritz SAH, Kisdi É, Meszéna G, Metz JAJ (2004). Adaptive dynamics of speciation: Ecological underpinnings. In Adaptive Speciation, eds. Dieckmann U, Doebeli M, Metz JAJ \& Tautz D, pp.54-75. Cambridge, UK: Cambridge University Press

Gomulkiewicz R \& Holt RD (1995). When does evolution by natural selection prevent extinction? Evolution 49:201-207

Gould SJ \& Lewontin RC (1979). The spandrels of San Marco and the Panglossian paradigm: A critique of the adaptationist programme. Proceedings of the Royal Society of London B 205:581-598

Guckenheimer J \& Holmes P (1997). Nonlinear Oscillations, Dynamical Systems, and Bifurcations of Vector Fields. Third Edition. New York, NY, USA: Springer-Verlag 
Gyllenberg M \& Metz JAJ (2001). On fitness in structured metapopulations. Journal of Mathematical Biology 43:545-560

Gyllenberg M \& Parvinen K (2001). Necessary and sufficient conditions for evolutionary suicide. Bulletin of Mathematical Biology 63:981-993

Gyllenberg M, Parvinen K \& Dieckmann U (2002). Evolutionary suicide and evolution of dispersal in structured metapopulations. Journal of Mathematical Biology 45:79-105

Haldane JBS (1932). The Causes of Evolution. London, UK: Harper

Hamilton WD (1967). Extraordinary sex ratios. Science 156:477-488

Hastings A (1980). Disturbance, coexistence, history, and competition for space. Theoretical Population Biology 18:363-373

Heino M, Metz JAJ \& Kaitala V (1997). Evolution of mixed maturation strategies in semelparous life histories: The crucial role of dimensionality of feedback environment. Philosophical Transactions of the Royal Society of London B 352:647-1655

Heino M, Metz JAJ \& Kaitala V (1998). The enigma of frequency-dependent selection. Trends in Ecology and Evolution 13:367-370

Jablonski D (1993). The tropics as a source of evolutionary novelty through geological time. Nature 364:142-144

Jansen VAA \& Mulder GSEE (1999). Evolving biodiversity. Ecology Letters 2:379-386

Johnson PA, Hoppensteadt FC, Smith JJ \& Bush GL (1996). Conditions for sympatric speciation: A diploid model incorporating habitat fidelity and non-habitat assortative mating. Evolutionary Ecology 10:187-205

Kimura M (1965). A stochastic model concerning maintenance of genetic variability in quantitative characters. Proceedings of the National Academy of Sciences of the USA 54:731-735

Kirkpatrick M (1982). Sexual selection and the evolution of female mating preferences. Evolution 36:1-12

Kirkpatrick M (1996). Genes and adaptation: A pocket guide to theory. In Adaptation, eds. Rose MR \& Lauder GV, pp. 125-128. San Diego, CA, USA: Academic Press

Kisdi É \& Meszéna G (1993). Density-dependent life-history evolution in fluctuating environments. In Adaptation in a Stochastic Environment, eds. Yoshimura J \& Clark C, p. 26-62, Lecture Notes in Biomathematics Vol. 98. Berlin, Germany: Springer-Verlag

Kozlowski J (1993). Measuring fitness in life history studies. Trends in Ecology and Evolution 8:84-85

Kozlowski J \& Wiegert RG (1986). Optimal allocation of energy to growth and reproduction. Theoretical Population Biology 29:16-37

Kuznetsov YA (1995). Elements of Applied Bifurcation Theory. Berlin, Germany: SpringerVerlag

Law R (1979). Optimal life histories under age-specific predation. The American Naturalist 114:399-417

Le Galliard JF, Ferrière R \& Dieckmann U (2003). The adaptive dynamics of altruism in spatially heterogeneous populations. Evolution 57:1-17

Levins R (1962a). Theory of fitness in a heterogeneous environment. I. The fitness set and adaptive function. The American Naturalist 96:361-373

Levins R (1962b). Theory of fitness in a heterogeneous environment. II. Developmental flexibility and niche selection. The American Naturalist 97:74-90

Levins R (1968). Evolution in Changing Environments. Princeton, NJ, USA: Princeton University Press 
Lewontin RC (1979). Fitness, survival, and optimality. In Analysis of Ecological Systems, eds. Horn DJ, Stairs GR \& Mitchell RD, pp. 3-22. Columbus, OH, USA: Ohio State University Press

Lewontin RC (1987) The shape of optimality. In The Latest on the Best, ed. Dupre J, pp. 151-159. Cambridge, MA, USA: MIT Press

Losos JB (1996). Ecological and evolutionary determinants of the species-area relation in Caribbean anoline lizards. Philosophical Transactions of the Royal Society of London B 351:847-854

Losos JB \& Schluter D (2000). Analysis of an evolutionary species-area relationship. $\mathrm{Na}$ ture 408:847-850

MacArthur RH \& Wilson EO (1967). The Theory of Island Biogeography. Princeton, NJ, USA: Princeton University Press

Matessi C, Gimelfarb A \& Gavrilets S (2001). Long term buildup of reproductive isolation promoted by disruptive selection: How far does it go? Selection 2:41-64

Mathias A \& Kisdi É (2002). Adaptive diversification of germination strategies. Proceedings of the Royal Society of London B 269:151-155

Matsuda H (1985). Evolutionarily stable strategies for predator switching. Journal of Theoretical Biology 115:351-366

Matsuda H \& Abrams PA (1994a). Runaway evolution to self-extinction under asymmetrical competition. Evolution 48:1764-1772

Matsuda H \& Abrams PA (1994b). Timid consumers - self-extinction due to adaptive change in foraging and anti-predator effort. Theoretical Population Biology 45:76-91

May RM \& Nowak MA (1994). Superinfection, metapopulation dynamics, and the evolution of diversity. Journal of Theoretical Biology 170:95-114

May RM \& Oster GF (1976). Bifurcations and dynamic complexity in simple ecological models. The American Naturalist 110:573-599

Maynard Smith J (1966). Sympatric speciation. The American Naturalist 100:637-650

Maynard Smith J (1982). Evolution and the Theory of Games. Cambridge, UK: Cambridge University Press

Maynard Smith J \& Price GR (1973). Logic of animal conflict. Nature 246:15-18

Maynard Smith J, Burian R, Kauffman S, Alberch P, Campbell J, Goodwin B, Lande R, Raup D \& Wolpert L (1985). Developmental constraints and evolution. Quarterly Review of Biology 60:265-287

Mayr E (1963). Animal Species and Evolution. Cambridge, MA, USA: Harvard University Press

Mayr E (1982). The Growth of Biological Thought: Diversity, Evolution, and Inheritance. Cambridge, MA, USA: The Belknap Press of Harvard University Press

Meszéna G, Kisdi É, Dieckmann U, Geritz SAH \& Metz JAJ (2001). Evolutionary optimisation models and matrix games in the unified perspective of adaptive dynamics. Selection 2:193-210

Metz JAJ \& Gyllenberg M (2001). How should we define fitness in structured metapopulation models? Including an application to the calculation of evolutionarily stable dispersal strategies. Proceedings of the Royal Society of London B 268:499-508

Metz JAJ, Nisbet RM \& Geritz SAH (1992). How should we define fitness for general ecological scenarios. Trends in Ecology and Evolution 7:198-202

Metz JAJ, Geritz SAH, Meszéna G, Jacobs FJA \& Van Heerwaarden JS (1996a). Adaptive dynamics, a geometrical study of the consequences of nearly faithful reproduction. In 
Stochastic and Spatial Structures of Dynamical Systems, eds. Van Strien SJ \& Verduyn Lunel SM, pp. 183-231. Amsterdam, Netherlands: North-Holland

Metz JAJ, Mylius SD \& Diekmann O (1996b). When Does Evolution Optimize? On the Relation between Types of Density Dependence and Evolutionarily Stable Life History Parameters. IIASA Working Paper WP-96-004. Laxenburg, Austria: International Institute for Applied Systems Analysis

Meyer A (1993). Phylogenetic relationships and evolutionary processes in east African cichlid fishes. Trends in Ecology and Evolution 8:279-284

Mooney HA \& Cleland EE (2001). The evolutionary impact of invasive species. Proceedings of the National Academy of Sciences of the USA 98:5446-5451

Mooney HA \& Hobbs RJ, eds. (2000). Invasive Species in a Changing World. Washington, DC, USA: Island Press

Mouquet N, Mulder GSEE, Jansen VAA \& Loreau M (2001). The properties of competitive communities with coupled local and regional dynamics. In Dispersal, eds. Clobert J, Danchin E, Dhondt AA \& Nichols JD, pp. 311-326. Oxford, UK: Oxford University Press

Myers N \& Knoll AH (2001). The biotic crisis and the future of evolution. Proceedings of the National Academy of Sciences of the USA 98:5389-5392

Mylius SD \& Diekmann O (1995). On evolutionarily stable life histories, optimization and the need to be specific about density dependence. Oikos 74:218-224

Nowak MA \& May RM (1994). Superinfection and the evolution of parasite virulence. Proceedings of the Royal Society of London B 255:81-89

Nowak M \& Sigmund K (1989). Oscillations in the evolution of reciprocity. Journal of Theoretical Biology 137:21-26

Parvinen K \& Dieckmann U. Evolutionary suicide. In Elements of Adaptive Dynamics, eds. Dieckmann U \& Metz JAJ. Cambridge, UK: Cambridge University Press. In press

Parvinen K \& Dieckmann U. Even frequency-independent selection can cause selfextinction. Unpublished

Pásztor L, Meszéna G \& Kisdi É (1996). $R_{0}$ or $r$ : A matter of taste? Journal of Evolutionary Biology 9:511-518

Perrings C, Williamson M, Barbier EB, Delfino D, Dalmazzone S, Shogren J, Simmons P \& Watkinson A (2002). Biological invasion risks and the public good: An economic perspective. Conservation Ecology 6:1

Renault $\mathrm{O}$ \& Ferrière R. Population size and persistence under demographic stochasticity and overcompensatory density dependence. Unpublished

Roff DA (1992). The Evolution of Life Histories: Theory and Analysis. New York, NY, USA: Chapman \& Hall

Roff DA (2002). Life History Evolution. Sunderland, MA, USA: Sinauer Associates Inc.

Rosenzweig ML (1978). Competitive speciation. Biological Journal of the Linnean Society 10:275-289

Rosenzweig ML (1995). Species Diversity in Space and Time. Cambridge, UK: Cambridge University Press

Rosenzweig ML (2001). Loss of speciation rate will impoverish future diversity. Proceedings of the National Academy of Sciences of the USA 98:5404-5410

Roughgarden J (1979). Theory of Population Genetics and Evolutionary Ecology: An Introduction. New York, NY, USA: Macmillan

Roughgarden J (1995). Anolis Lizards of the Caribbean. Oxford, UK: Oxford University Press 
Schliewen UK, Tautz D \& Pääbo S (1994). Sympatric speciation suggested by monophyly of crater lake cichlids. Nature 368:629-632

Schluter D (2000). The Ecology of Adaptive Radiation. Oxford, UK: Oxford University Press

Sinervo B \& Lively CM (1996). The rock-paper-scissors game and the evolution of alternative male strategies. Nature 380:240-243

Sinervo B, Svensson E \& Comendant T (2000). Density cycles and an offspring quantity and quality game driven by natural selection. Nature 406:985-988

Soulé ME (1980). Thresholds for survival: Maintaining fitness and evolutionary potential. In Conservation Biology: An Evolutionary-Ecological Perspective, eds. Soulé ME \& Wilcox BA, pp. 151-170. Sunderland, MA, USA: Sinauer Associates Inc.

Stearns SC (1992). The Evolution of Life Histories. Oxford, UK: Oxford University Press

Stearns SC \& Koella JC (1986). The evolution of phenotypic plasticity in life-history traits - predictions of reaction norms for age and size at maturity. Evolution 40:893-913

Taylor PD (1989). Evolutionary stability in one-parameter models under weak selection. Theoretical Population Biology 36:125-143

Tilman D (1994). Competition and biodiversity in spatially structured habitats. Ecology 75:2-16

Tilman D \& Lehman CL (2001). Human-caused environmental change: Impacts on plant diversity and evolution. Proceedings of the National Academy of Sciences of the USA 98:5433-5440

Tilman D, May RM, Lehman CL \& Nowak MA (1994). Habitat destruction and the extinction debt. Nature 371:65-66

Van Tienderen PH \& de Jong G (1986). Sex-ratio under the haystack model - polymorphism may occur. Journal of Theoretical Biology 122:69-81

Williamson M (1996). Biological Invasions. London, UK: Chapman \& Hall

Wright S (1931). Evolution in Mendelian populations. Genetics 16:97-159

Wright $S$ (1932). The roles of mutation, inbreeding, crossbreeding and selection in evolution. Proceedings of the 6th International Congress of Genetics 1:356-366

Wright S (1967). Surfaces of selective value. Proceedings of the National Academy of Sciences of the USA 102:81-84

Wright S (1969). Evolution and the Genetics of Populations, Volume 2. Chicago, IL, USA: University of Chicago Press

Wright S (1988). Surfaces of selective value revisited. The American Naturalist 131:115123

Yodzis P (1989). Introduction to Theoretical Ecology. New York, NY, USA: Harper \& Row 